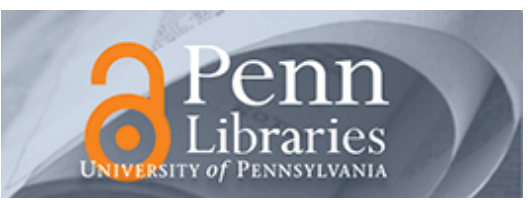

University of Pennsylvania

ScholarlyCommons

Departmental Papers (ESE)

Department of Electrical \& Systems Engineering

$12-2013$

\title{
Convergence of Bayesian Histogram Filters for Location Estimation
}

\author{
Avik De \\ University of Pennsylvania
}

Alejandro Ribeiro

University of Pennsylvania

William Moran

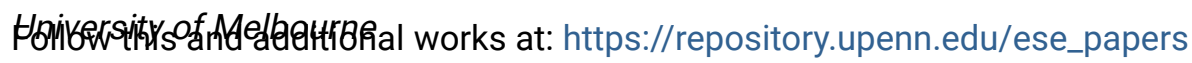

rPertEoflkbeliterectuékal and Computer Engineering Commons, and the Systems Engineering Commons University of Pennsylvania, kod@seas.upenn.edu

\section{Recommended Citation}

Avik De, Alejandro Ribeiro, William Moran, and Daniel E. Koditschek, "Convergence of Bayesian Histogram Filters for Location Estimation", . December 2013.

BibTeX entry

@inproceedings\{paper:de_histogram_2013, author = \{Avik De, Alejandro Ribeiro, William Moran and Daniel E. Koditschek $\}$, title $=\{$ Convergence of Bayesian Histogram Filters for Location Estimation $\}$, booktitle $=\{$ Proceedings of the 2013 IEEE Intl. Conference on Decision and Control $\}$, month $=\{$ December $\}$, year $=\{2013\}\}$

This work was supported by AFOSR MURI FA9550-10-1-0567.

Copyright 2013 IEEE. Reprinted from Proceedings of the 2013 IEEE Intl. Conference on Decision and Control.

This material is posted here with permission of the IEEE. Such permission of the IEEE does not in any way imply IEEE endorsement of any of the University of Pennsylvania's products or services. Internal or personal use of this material is permitted. However, permission to reprint/republish this material for advertising or promotional purposes or for creating new collective works for resale or redistribution must be obtained from the IEEE by writing to pubs-permissions@ieee.org. By choosing to view this document, you agree to all provisions of the copyright laws protecting it.

This paper is posted at ScholarlyCommons. https://repository.upenn.edu/ese_papers/660

For more information, please contact repository@pobox.upenn.edu. 


\title{
Convergence of Bayesian Histogram Filters for Location Estimation
}

\begin{abstract}
We prove convergence of an approximate Bayesian estimator for the (scalar) location estimation problem by recourse to a histogram approximant. We exploit its tractability to present a simple strategy for managing the tradeoff between accuracy and complexity through the cardinality of the underlying partition. Our theoretical results provide explicit (conservative) sufficient conditions under which convergence is guaranteed. Numerical simulations reveal certain extreme cases in which the conditions may be tight, and suggest that this procedure has performance and computational efficiency favorably comparable to particle filters, while affording the aforementioned analytical benefits. We posit that more sophisticated algorithms can make such piecewise-constant representations similarly feasible for very high-dimensional problems.

For more information: Kod*Lab

Disciplines

Electrical and Computer Engineering | Engineering | Systems Engineering

Comments

BibTeX entry

@inproceedings\{paper:de_histogram_2013, author = \{Avik De, Alejandro Ribeiro, William Moran and Daniel E. Koditschek $\}$, title $=\{$ Convergence of Bayesian Histogram Filters for Location Estimation $\}$, booktitle $=$ \{Proceedings of the 2013 IEEE Intl. Conference on Decision and Control $\}$, month $=\{$ December $\}$, year $=$ $\{2013\}\}$

This work was supported by AFOSR MURI FA9550-10-1-0567.

Copyright 2013 IEEE. Reprinted from Proceedings of the 2013 IEEE Intl. Conference on Decision and Control.

This material is posted here with permission of the IEEE. Such permission of the IEEE does not in any way imply IEEE endorsement of any of the University of Pennsylvania's products or services. Internal or personal use of this material is permitted. However, permission to reprint/republish this material for advertising or promotional purposes or for creating new collective works for resale or redistribution must be obtained from the IEEE by writing to pubs-permissions@ieee.org. By choosing to view this document, you agree to all provisions of the copyright laws protecting it.
\end{abstract}




\title{
A Bioinspired Dynamical Vertical Climbing Robot
}

\author{
Goran A. Lynch, Jonathan E. Clark, Pei-Chun Lin, and Daniel E. Koditschek
}

\begin{abstract}
This paper describes the inspiration, design, analysis, implementation of and experimentation with the first dynamical vertical climbing robot. Biologists have proposed a pendulous climbing model that abstracts remarkable similarities in dynamic wall scaling behavior exhibited by radically different animal species. We study numerically a version of that pendulous climbing template dynamically re-scaled for applicability to utilitarian payloads with conventional electronics and actuation. This simulation study reveals that the incorporation of passive compliance can compensate for an artifact's poorer power density and scale disadvantages relative to biology. However the introduction of additional dynamical elements raises new concerns about stability regarding both the power stroke and limb coordination that we allay via mathematical analysis of further simplified models. Combining these numerical and analytical insights into a series of design prototypes, we document the correspondence of the various models to the variously scaled platforms and report that our approximately two kilogram platform climbs dynamically at vertical speeds up to 1.5 bodylengths per second. In particular, the final $2.6 \mathrm{~kg}$ final prototype climbs at an average steady state speed of $0.66 \mathrm{~m} / \mathrm{s}$ against gravity on a carpeted vertical wall, in rough agreement with our various models' predictions.
\end{abstract}

\section{INTRODUCTION}

Past climbing robots have been slow and in most instances restricted to targeted surfaces where specific attachment mechanisms such as suction and electromagnetic adhesion can be brought to bear [5], [6]. Over the last decade robots have been built that are capable of more broadly effective attachment, for example by means of footholds $[7,8,9]$ or vectored thrust $[10$, 11]. The last few years have also seen the revival [12], [13, 14] of rimless wheels with sticky toes [15], [16] to intermittently "roll" up smooth walls and the development of novel spined and sticky feet for attachment to smooth surfaces such as concrete and brick $[17,18]$. Despite the dramatic improvement of attachment technologies, robotic climbers can still generally progress only slowly up vertical surfaces.

The unremitting cost of work against gravity seems significantly less constraining in the animal kingdom which boasts a variety of species that can dynamically speed their way up vertical environments surfaced in a broad variety of materials, textures, and geometries. Recent biomechanical studies of small, agile, climbing animals reveal a striking similarity in

Some of the component pieces of the present account have been offered in preliminary form in various conferences: [1] (initial actuation analysis); [2] (initial platform design); [3] (initial empirical climbing results); and [4] (robot controller design).

G. A. Lynch, and D. E. Koditschek are with GRASP Laboratory, University of Pennsylvania Department of Electrical and Systems Engineering, Philadelphia, PA, USA.

J. E. Clark is with the Department of Mechanical Engineering, FAMUFSU College of Engineering, 2525 Pottsdamer Str. A-229, Tallhassee, FL, 32310, USA e-mail: clarkj@eng.fsu.edu.

P. C. Lin is with the Department of Mechanical Engineering, National Taiwan University, Taipei, Taiwan. locomotion dynamics that belies stark differences in attachment mechanisms, morphology, and phylogeny [19]. These unexpectedly common patterns can be abstracted in a simple numerical model that raises the prospect of a "template" [20] for dynamical climbing analogous to the ubiquitous SpringLoaded Inverted Pendulum (SLIP) model [21, 22, 23] in sagittal, level-ground runners and Lateral-Leg Spring (LLS) [24] in sprawled level ground (horizontal) runners. In this paper we explore the value and applicability of this new biological climbing template to the domain of robotics. We aim to build a fast, agile climbing robot capable of dynamical operation across a broad variety of scansorial regimes, and we wish to test the proposition that adapting this new biological template will prove both viable and effective to that end. This paper takes a first comprehensive step toward doing so by introducing a low degree of freedom electromechanical design that instantiates the template and exhibits dynamical climbing, along with a family of mathematical models used to inform and guide this design that pursues scaling arguments and actuation analysis to draw the abstract animal model [19] toward viable realization as an engineered artifact.

Since the initial presentation of our biologically inspired dynamic climber [3], a number of other platforms have been developed that also explicitly exploit their dynamics in climbing, including: CMU's DynaClimber which braces against parallel vertical walls to generate dynamic upward locomotion $[25,26]$, the newest RiSE robot which ascends cylindrical vertical surfaces rapidly (up to $22 \mathrm{~cm} / \mathrm{s}$ ) using a pseudobound gait [27], and ROCR [28], a T-shaped climber which ascends by exciting a pendular rotation using an actuated tail. While the variety of these designs clearly indicate the interest in, and potential utility of, dynamic climbing, the research described here offers several several advantages that stem from the biological inspiration behind our work. These include: animal-like climbing speeds (over one body-length per second), rapid recovery from missed footholds, and the prospect for systematic comparison with and gradual incorporation of accumulating features of animal climbing. Chief among these intriguing future directions of inquiry are: (i) the role of sprawled attachment; (ii) the consequent prominence of lateral forces in dynamic climbing; and (iii) the organization of level-to-vertical transitions in dynamic locomotion. ${ }^{1}$

The paper is organized according to the following progression of bioinspired concepts, engineering implementation challenges, our proposed solutions, their partial mathematical verification, and consequent empirical validation. Section II briefly reviews the motivating bioinspiration, presenting the original Full-Goldman template model [19] and recounting

${ }^{1}$ Mounting evidence [19] suggests that animals switch from outward to inward lateral force generation when they switch from horizontal to vertical running. 
its origins in animal climbing studies. Section III motivates and uses scaling arguments to explore the consequences of an order of magnitude increase in length. This increase is necessitated by the goal of an engineered artifact that might be realized as a utilitarian robot in the near term using present commercially available components. However, the template model [19], when scaled up to the prescribed utilitarian mass and length, entails a power density beyond the range of contemporary commercial actuators.

We consider this problem in Section IV, and after reviewing the principal design alternatives, settle on an actuation scheme that addresses the power density deficit in two related ways. First, we place a spring in parallel with the motor as a means of smoothing its power output over the stride, thereby reducing the peak power requirement to the point of plausible recourse to commercial off the shelf (hereafter, COTS) actuators. Second, we abandon the assumption that the resulting power train will simply deliver the prescribed leg length trajectory over time in favor of a force control policy. Recognizing our true affordance is in reality limited to prescribing motor terminal voltages, such a policy seeks to extract as much work against gravity as the motors can deliver.

The introduction of this more realistic explicit actuation model complicates the template by exposing it to new "internal" dynamics in two ways. First, the body mass now experiences ground reaction forces through the coupled parallel spring-motor dynamics raising the possibility that the resulting new (hybrid) forced spring-mass system might fail to converge to the period-1 climbing gait demonstrated by the template. Second, since the actuators' phases are no longer prescribed but emerge through their dynamical interactions with the mass, we must introduce an explicit coordination scheme to keep them pulling in alternation rather than bunching together in a phase-locked ("pronking") mode.

Accordingly, in Section V we study the simplest useful mathematical representations of isolated versions of these new sources of climbing dynamics to insure that our solutions do not introduce stability problems - at least in decoupled configurations. In Section V-A, we consider a hypothetical perfectly coordinated climber and examine in isolation the power train dynamics: the actuator and passive spring coupled in parallel to a one (purely vertical) degree of freedom climbing mass. After deriving the model's return map, we prove formally that it climbs with the desired stable, period-1 gait over a broad regime in parameter space relevant to the range of physically interesting designs. Then, in Section V-B, we present a succession of two force controlled actuator coordination strategies. We reject an initial hybrid force/mirror-law scheme following early empirical tests (relevant data reported in Section VI-B), then replace it with a "self-exciting" force control scheme that seems likelier to extract more of the actuators' work against gravity. We examine an abstracted version of this coordination scheme and prove mathematically the stability of the desired anti-phase limit cycle.

Informed by these numerical and mathematical analyses, we proceed in Section VI to relate their impact upon the actual physical implementation, and document its efficacy by presenting data obtained from a series of actual climbing

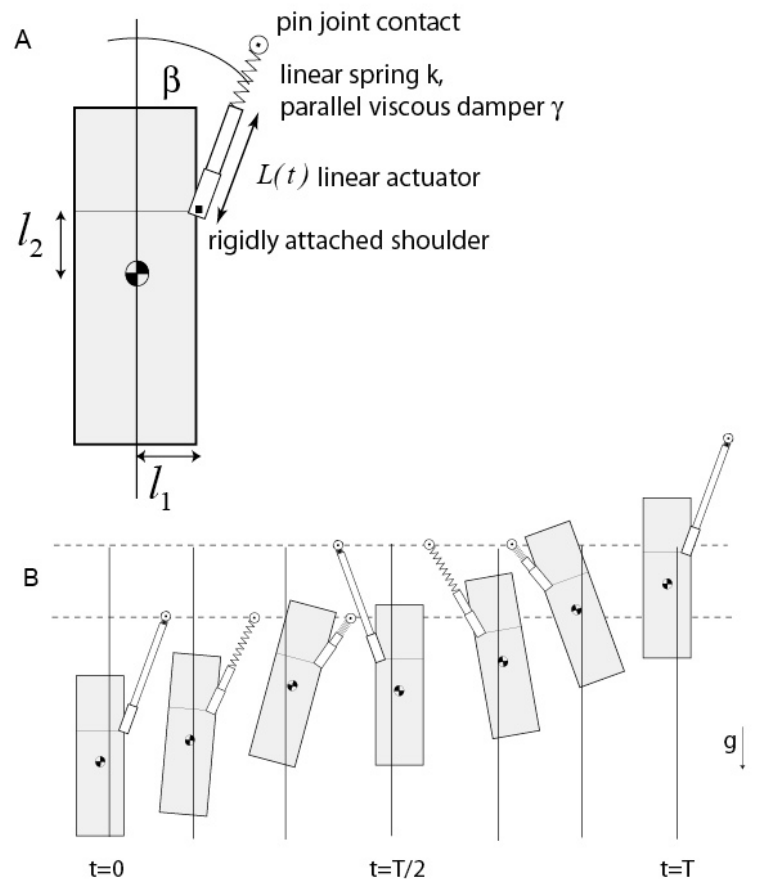

Fig. 1. The Full-Goldman (FG) dynamic template for climbing, with $g$ as the direction of gravity. The two degree of freedom model that generates the template climbing dynamics shown in Fig. 2C. (A) Schematic of the model. (B) Schematic of the motion of the model during two steps. The extension of the spring has been exaggerated for clarity. Reproduced with permission from [19].

experiments. An initial design achieved in physical hardware yielded a working version of the template inspired climber - historically, the first dynamical vertical climbing robot. However, this preliminary design, while dynamically similar to the template, did not achieve the targeted climbing speed: the foregoing numerical and mathematical analyses suggested a series of design modifications which, when implemented now exhibited the desired dynamics but with a far more favorable climbing speed.

We conclude by commenting on some of the broader issues associated with robot climbers, and discuss future work including limb coupling dynamics, energetics, stability, and adaptation to a more utilitarian polypedal morphology.

\section{Biological Origins of the Climbing Template}

Organisms as diverse as arthropods and vertebrates use differing limb number, attachment mechanism and body morphology to achieve performance on vertical substrates that rivals level ground running, hence it is natural to anticipate that diverse animals would develop correspondingly divergent climbing strategies. Surprisingly, Goldman et al. [19] have discovered common dynamics in quite different rapidly climbing organisms, a cockroach and a gecko. Perhaps equally surprising, neither climbs straight up a vertical flat wall. Both organisms generate large, alternating lateral forces during climbs over 4 bodylengths per second that produce substantial changes in lateral as well as fore-aft velocity $[19,29]$. 
A specific model which generates the template dynamics of vertical climbing is shown in Fig. 1A and a schematic of its motion in Fig. 1B. The model consists of a rigid body that is pulled upward and side-to-side through the action of a spring in series with a linear actuator.

As shown in Fig. 1, in the first step with the right leg, at touchdown $(t=0)$ the right actuator is maximally extended, and the spring is relaxed with zero rest length. Touchdown is created by establishment of a rotationally free pin joint with the wall. As the actuator length $L(t)$ decreases, the spring in the leg extends, the foot freely pivots about the point of contact and the center of mass (COM) is translated vertically and laterally. The stance foot is released at a fixed point near maximum compression in the leg cycle and the process repeats for the left leg. The actuator changes length sinusoidally such that

$$
L(t)=\frac{l_{s}}{2}(1+\sin (2 \pi f t))+L_{0},
$$

where $l_{s}$ is the step length, $L_{0}$ is the retracted length of the leg, and $f$ is the stride frequency. The solid vertical line in each panel indicates the fixed lateral position about which the center of mass laterally oscillates. The angular excursion of the body and extension of the spring are exaggerated for clarity. Actual angular excursion of the body relative to vertical is approximately $\pm 3^{\circ 2}$. The model was coded and integrated in the Working Model 2D (Design Simulation Technologies, Inc) simulation environment.

The forces and resulting center of mass velocities generated by this position-based control of the actuators on the model are shown in Fig. 2 and agree well with the published patterns measured in cockroaches and geckos [19]. ${ }^{3}$ The representative magnitude and phasing of forces and center of mass velocities was found after systematic variation of system parameters[19].

We now proceed to discuss the sequence of design choices leading to the construction of a robot which anchors this template. ${ }^{4}$

\section{SCALING OF TEMPlate}

While the Full-Goldman template was designed to model the locomotion of animals with masses on the order of $2 \mathrm{~g}$, present day climbing robots that have achieved $[5,28,31]$ or seem close $[8,11,18]$ to utilitarian realization are all several $(2-3)$ orders of magnitude larger and it is these existing designs (mostly quasi-static in operation) that will

\footnotetext{
${ }^{2}$ The template does not brachiate or actively swing in a pendulous manner to raise its center of mass. Rather, sprawl angle, when teamed with an adequately rapid stride frequency, minimizes angular deviation as evidenced by the minimal angular excursion demonstrated by the template.

${ }^{3}$ The parameters used to generate Fig. $2 \mathrm{C}$ were body mass $=2 \mathrm{~g}$, body dimensions $=4 \mathrm{~cm} \times 0.95 \mathrm{~cm}, l_{1}=0.71 \mathrm{~cm}, l_{2}=0.84 \mathrm{~cm}, \beta=10$ degrees, $L_{0}=1.54 \mathrm{~cm}, z=0.6, k=6 \mathrm{Nm}^{-1}, \gamma=0.09 N-s m^{1}, f=9 \mathrm{~Hz}$. The attachment duty factor in the model is 0.46 . The rigid body has a moment of inertia of $8 \times 10^{-7} \mathrm{~kg}-\mathrm{m}^{2}$, the order of magnitude of cockroaches $\left(2 \times 10^{-7} \mathrm{~kg}-\mathrm{m}^{2}\right)$ [30].

${ }^{4}$ Dynoclimber's morphology as depicted in Fig. 12 bears more than passing kinematic resemblance to the template of Fig. 1. Anchoring that template in a robot with more legs is certainly possible, since the model was constructed to help understand the locomotion strategies of 4 and 6 legged animals. The specific morphology and controller for such a robot would supply a net force applied to the robots center of mass which matched the force applied to the center of mass of the template.
}
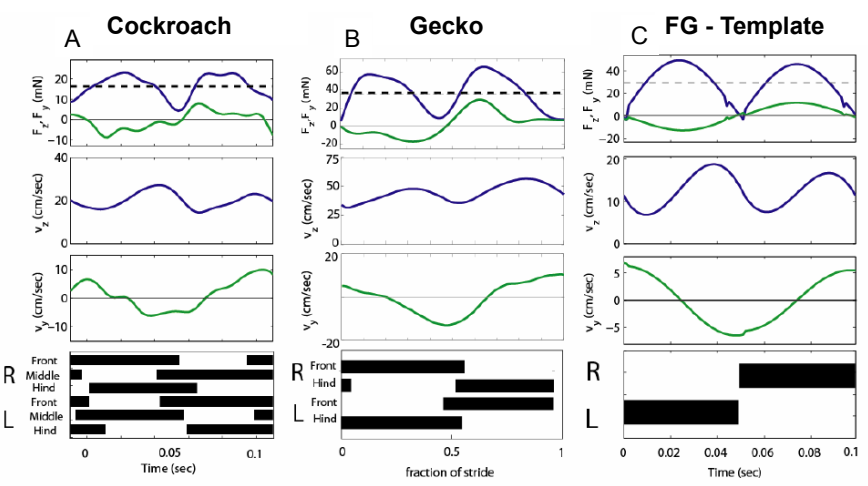

Fig. 2. Force, vertical velocity $\left(V_{z}\right)$, lateral velocity $\left(V_{y}\right)$, and foot fall patterns for the cockroach, gecko, and the Full-Goldman template. Broken lines indicate body weight. Data are shown for a normalized stride, with black bars representing foot contact. In each force plot $F_{z}$ is the magnitude in the vertical direction and $F_{y}$ is in lateral direction. Reproduced with permission from [19].

offer the most immediate basis for comparing the relative value of dynamical climbing in robotics. More pragmatically, it is only in recent years that novel technologies have emerged permitting the construction of legged robots at the small (1$10 \mathrm{~g}$ ) scale ( e.g., [32] presents a $16 \mathrm{~g}$ legged runner). We would likely have to rely on remote-control operation (as did the authors of [32]), and eliminate any local control. The lack of local processing would not allow for high-bandwidth feedback control to be conducted locally. Moreover, we expect nearterm future dynamic climbers to be faced with complex terrain navigation decisions which mandate on-board intelligence. DynoClimber, as a dynamics testbed for such platforms, is designed at a scale for which computational payloads are not prohibitively large.

Given the contemporary prevalence of kg-scale climbing machines and the distracting difficulties of constructing a robot at the template's $2 \mathrm{~g}$ mass, we instead scale the physical parameters of the template to arrive at a climbing model which demonstrates dynamically similar climbing behavior with a more easily realizable size and mass. Notably, as we demonstrate in this section, any increase in climber size necessitates an increase in power density to maintain dynamic similarity. Since COTS motor power density is roughly constant over variations in motor size (as can qualitatively be discerned from catalogues [33]), for the power outputs which we are able to comfortably sustain with familiar technology, the approximate upper mass and speed limit at which the robot can climb dynamically is kilogram-scale. In Section IV-B, we demonstrate that we require passive-elastic energy storage springs to achieve dynamic locomotion with a $2 \mathrm{~kg}$ robot, indicating that we are near the maximum size at which dynamic climbing according to the Full-Goldman template can be achieved.

With a target mass of $2 \mathrm{~kg}$ for DynoClimber, we shift attention to the questions of scaling: what we mean by dynamically similar behavior and how it can be achieved. Guided by [34], we pursue "dynamic similarity," meaning that displacements, times, and forces of the scaled system should be simple, scaled versions of the original. By preserving the ratios between 
each of the natural and driven frequencies within the system, dynamic similarity is achieved [34] and stability properties remain unchanged [35]: in particular, our scaling procedure preserves the template's Strouhal number, as well as its Froude number, used to characterize scale-independent running speed $[36,37]$. We use the term dynamically similar (in the sense of [38]) to denote the additional mathematical consequence of such scaling relationships that guarantee formal conjugacy of all resulting dynamical models: in particular the stability properties of all steady state behaviors are preserved across all models.

We now compute the scale factors which expand the $2 g$ template into a $2 \mathrm{~kg}$, dynamically similar variant. We assume that mass varies as the cube of length, and therefore scale all body dimensions (including stroke length) linearly by a factor, $\alpha_{L}=10$, resulting in the desired mass scaling of $\alpha_{L}^{3}=1000$. Recalling that rotational inertia for a point mass at a distance $r$ from the pivot point is $m \cdot r^{2}$, the scaled template's rotational inertia is, as a result of the length and mass scaling, increased by a factor of $\alpha_{L}^{3} \cdot \alpha_{L}^{2}=\alpha_{L}^{5}$.

The central target of our scaling procedure is the maintenance of frequency ratios within the system, one of which is established by the simple scale factors we have already determined: the climber's pendular rotation frequency. The climber's body rotates about a pinned stance foot with a frequency of $\sqrt{\frac{m g L}{I}}$, where $m$ is mass, $g$ is gravity, $L$ the distance between the pivot point and the center of mass, and $I$ is the body's moment of inertia. Plugging in the known scale factors for these constants, we find that the natural frequency of the body scales as $\alpha_{\omega}=: \sqrt{\frac{\alpha_{L}^{3} * \alpha_{L}}{\alpha_{L}^{5}}}=\frac{1}{\sqrt{10}}=.316$.

The preservation of frequency ratios requires us to scale both the leg driving frequency and the wrist spring contraction frequency by the same $\alpha_{\omega}=.316$. The leg driving frequency is established by a controller and has no immediate physical design consequences; we simply reduce the driven frequency from $9 \mathrm{~Hz}$ to $2.85 \mathrm{~Hz}$.

During any given stance mode, the climber's wrist spring supports the full mass of its body. When matched to a mass $m$, the spring oscillates with a frequency of approximately $\sqrt{\frac{k}{m}}$. In order to effect the correct scaling in the wrist contraction frequency, we must therefore alter the wrist spring constant. Thus, $\sqrt{\frac{\alpha_{k}}{\alpha_{L}^{3}}}=\alpha_{\omega}=\frac{1}{\sqrt{\alpha_{L}}}$ implies $\alpha_{k}:=\alpha_{L}^{2}=100$, and our wrist spring scale factor is determined.

Finally, we must choose a wrist spring damper. We choose a damping scale factor $\alpha_{b}$ which increases forces applied by the damper by the the same factor, $\alpha_{L}^{3}$, as other forces in the system. Since the force generated by a damper is $F_{d}=v \cdot b$, where $b$ is the damping constant, we must consider velocity as we determine the correct scaling for $b$. Realizing that system velocities scale by length times frequency $\alpha_{V}:=\alpha_{\omega} \cdot \alpha_{L}=$ $\sqrt{\alpha_{L}}=3.16$, we can now write the scaling law for $b$ :

$$
\alpha_{b}=: \frac{\alpha_{L}^{3}}{\alpha_{V}}=316 .
$$

The Strouhal number of the climber, given by $S t r:=\frac{\omega l}{V}$, is evidently preserved since $V$ scales as the product of $\omega$ and $l$. Our scaling also preserves the non-dimensional Froude number, $\operatorname{Fr}:=\frac{V}{\sqrt{g l}}$, since both $V$ and $\sqrt{l}$ scale as $\sqrt{\alpha_{L}}$.

With this scaling, dynamic similarity in the sense of [38] is maintained. However, dynamic similarity does not necessarily guarantee the preservation of other (scale dependent) performance metrics. For example, if we calculate speed using the widely used body-lengths per second (bl/s) metric, the template climbs at $4.5 \mathrm{bl} / \mathrm{s}$, and its scaled variant at only 1.4 $\mathrm{bl} / \mathrm{s}^{5}$
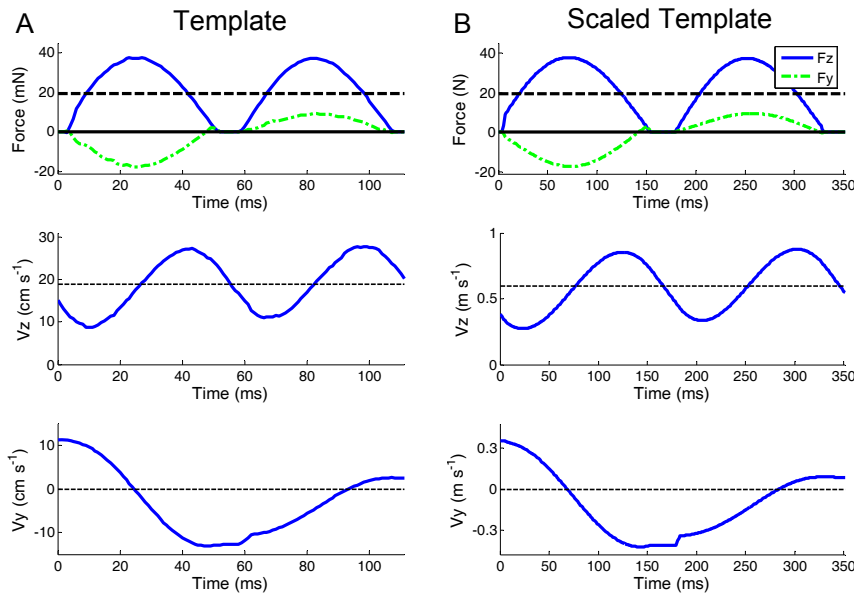

Fig. 3. Force, vertical velocity $\left(V_{z}\right)$, and lateral velocity $\left(V_{y}\right)$ for the animal template model and the scaled robot template. The heavy dashed horizontal lines represent the weight of the robot and the light dashed lines are the mean velocities. (Note the change in units for the scaled template).

Figure 3 presents a typical simulation output of steadystate climbing illustrating the effects of increased size on template behavior under the scaling model proposed above. The phasing of body motions and forces has been preserved, and simulations show that the scaled template's velocity and ground reaction force pattern matches prediction.

Biological actuators (muscles) differ from and are in many ways superior to current commercially available prime movers $[39,40]$. A nice overview - notably still current today - of the underlying physics and consequent operational properties of various candidate robotic actuators is given in [41]. From our perspective, the major limitation of commercially available actuation technology is that of power density. Our scaling assumptions require that an order of magnitude increase in length be supported by a three-fold increase in power density:

$$
\begin{aligned}
\alpha_{\frac{\text { power }}{\text { weight }}} & =\frac{F_{2 \mathrm{~kg}} \cdot V_{2 \mathrm{~kg}}}{m_{2 \mathrm{~kg}} \cdot g} \cdot \frac{m_{2 \mathrm{~g}} \cdot g}{F_{2 \mathrm{~g}} \cdot V_{2 \mathrm{~g}}}=\frac{\alpha_{L}^{3} \cdot \alpha_{V}}{\alpha_{L}^{3}} \\
& =\quad \alpha_{V}=3.16
\end{aligned}
$$

${ }^{5}$ Bodylengths/second scale as $\alpha_{V} / \alpha_{L}=1 / \sqrt{(10)}$. Although the Froude number for our scaled template is invariant, the absolute Froude value depends upon the characteristic length, $l$, of the template. This length is often described as the "leg length" or the distance from the hip to the foot, however it is not clear that this convention has the same effect in upright as in sprawled posture runners. For example, in our climber if the stroke length of the prismatic actuator is used as the characteristic leg length then the template and scaled climber have a characteristic speed of 0.73 , if the length from the "hip" to the foot is utilized, the Froude number becomes 0.45 , and if the body height is used it drops to 0.28 . Perhaps the intermediate value would be most appropriate for the purposes of comparison to biology, but regardless of which length is chosen the Froude number remains invariant to scale as long as the motions are dynamically similar. 
The increase required in power density has an intuitive explanation. With no energy sinks, vertical climbing speed is given by $\frac{\text { power }}{\text { force }}$, and at a constant velocity, the force applied is equal to the climber's weight. Thus, if the desired velocity increases, as it must to maintain dynamic similarity, the climber's power-to-weight ratio must increase correspondingly.

While we have chosen to restrict attention to conventionally amplified and driven commercial, brushed DC motors in this study, our experience, corroborated by the discussion in [41], is that all available alternatives can at best offer similar power density.

The reported record of actual power densities achieved in prior climbing and running robots varies greatly, but as a point of reference both the hexapedal RiSE [9] and RHex [42] robots have a specific power of about $10 \mathrm{~W} / \mathrm{kg}$ per tripod. Simulations at the roach scale show that each of the template's legs (each template leg is the equivalent of a tripod of cockroach legs) must achieve a peak power output of $6.3 \mathrm{~W}$ per kilogram of body mass. However, scaling the climber's body length by three orders of magnitude increases this power demand by a factor of 3.16 to $20 \mathrm{~W} / \mathrm{kg}$ per virtual leg (bringing total mechanical power output to $40 \mathrm{~W} / \mathrm{kg}$ ). Given that our scaled reference dynamics demand, at peak load, roughly twice the power endowment of previous legged robotic platforms, there are three alternatives: find (or design and build) higher power density actuators; reduce the peak power load; or settle for slower speeds. As described in IV, we take the middle course.

\section{Model Design Alterations for Realizability}

To build a robot which anchors the Full-Goldman template, we must modify the template's mass distribution, power transmission design, and approach to limb control. In this section we derive and discuss each of these changes with heavy reliance on simulation as a design tool. The resulting software model will be referred to as the "design simulation" throughout the sequel.

\section{A. Mass Distribution and Linkage Design}

To generate linear foot motion with a standard brushed rotational electric motor $^{6}$, we must build a transmission mechanism. Here we utilize a simple crank-slider mechanism (similar to the piston/crank used in automobile engines), as shown in Fig. 4. A principal advantage of this design is the unidirectional motor operation; the robot's motors do not need to change direction to reverse the direction of the feet.

To account for the mass of the attachment mechanism and transmission, we distribute some of the simulated climber's body mass to the legs. The total mass and footprint of the climber remains unchanged. A lateral degree of freedom and lateral compliance have been added to each of the hips to removes the kinematic singularity associated with double support phase. The stiffness and damping of these new lateral hips are set equal in magnitude to the wrist springs, but since

\footnotetext{
${ }^{6} \mathrm{We}$ choose to use rotational motors due to their high power density and extensive selection readily available from manufacturers such as Maxon[33].
}

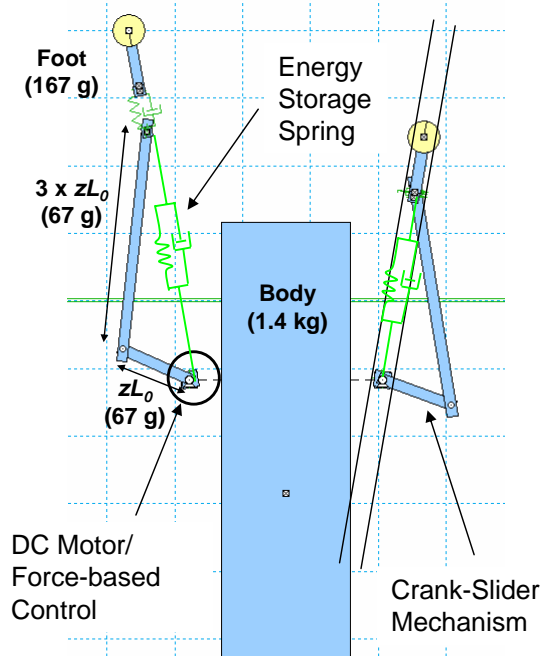

Fig. 4. Schematic of the crank-slider mechanism used to convert rotary (motor) output into linear motion. The relative lengths and masses of the links are indicated.

they are typically orthogonal to gravity, their deflection primarily occurs when both legs are attempting to simultaneously contract during incidental double support phases during the startup transient.

\section{B. Power Limitations}

As described in Section III, the power density required for a kilogram-scale bipedal dynamic climber $-20 \mathrm{~W} / \mathrm{kg}$ per leg - is double the power density achieved by former legged robots. Worse, preliminary simulations of a $2 \mathrm{~kg}$ crank-slider equipped climber indicate that the peak mechanical power output required from each motor exceeds $40 \mathrm{~W}$.

In this section we consider a number of methods which reduce the power output required of the climber's motors. Two prospective changes, shortening the stride length or decreasing the climbing frequency, prove effective in terms of reducing peak force, but affect the dynamics of climbing severely. Other, less dynamically disruptive choices include operating the motors with an accumulating "thermal debt", and mechanically coupling the robot's limbs. The approach which we eventually adopt in our climber is to utilize a passive dynamic element to store and return energy from the motor during the swing phase.

1) Simple Torque/Frequency Alterations: We consider the effects of, first, reducing actuation frequency and, second, shortening the crank moment arm. With either strategy, the naïve expectation is that if power applied is halved, climbing speeds will be halved as well.

The potential problem with altering the actuation frequency is that may disrupt the dynamics of climbing by producing a detrimental interaction with the body's pendular rotation. Simulations show that decreasing the stride frequency by a factor of two does result in stable motions, but there is much greater body swing during each stride. This dramatically increases the lateral forces seen at the feet and lateral velocity of the robot, as shown in Fig. 5A. This larger rotation reduces 

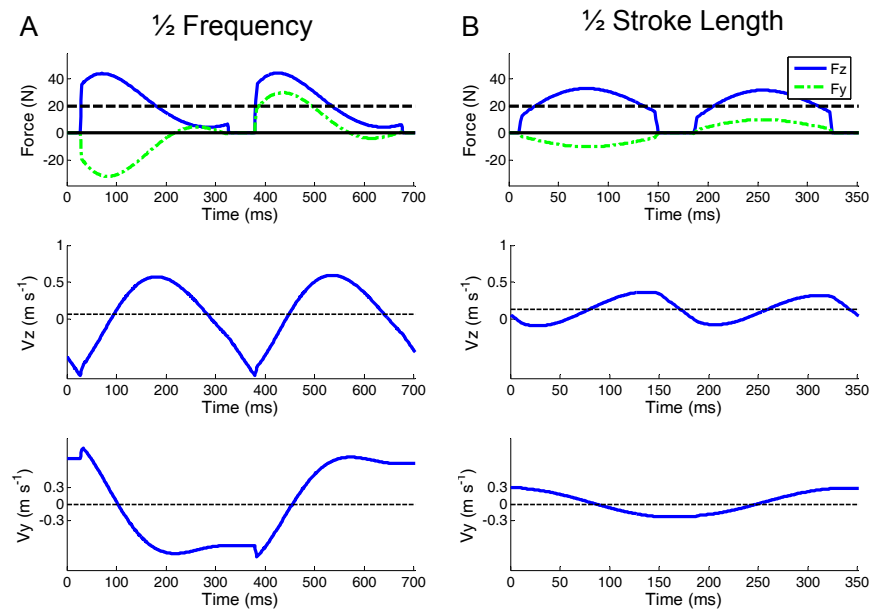

Fig. 5. Force, vertical and lateral velocity for two naïve approaches to reducing the power of the scaled template model. (A) Reduction of climbing frequency by one half and (B) shortening of the stroke length by one half. Note that the tick marks for force and velocity have been preserved from Fig. 3 to ease comparison.

the vertical component of each stride, dramatically (over 10x) reducing the upward speed of the climber. Peak required power, however, is reduced, in this case, from $40 \mathrm{~W}$ to $23 \mathrm{~W}$.

The second simple strategy, cutting the stride length in half by shortening the crank, only changes the pendular frequency of the body slightly, but this does not seem to significantly affect the stability of the gait. More significantly, as shown in Fig. 5B, the velocities of the climber with a reduced crank length are dramatically smaller than in the nominal case, with the net vertical velocity reduced to $20-25 \%$ of the scaled template's speed. The peak required power, however, is reduced to $18 \mathrm{~W}$.

In both cases we see that using naive approaches to reduce the peak power required from the actuator results in serious compromises in performance relative to the scaled template simulation. Thus, we pursue more sophisticated methods of coping with limited actuator power.

2) Thermal debt and Limb Coupling: One such method is to intermittently operate the motors above their continuously sustainable current limits. The motors may be "overrun" for short periods at the expense of producing more heat than can be dissipated. Too much time above the continuous current rating and the motors will eventually overheat and sustain irreversible physical damage. Thus, "thermal borrowing" can be used for short periods if the mean power draw is low enough. Indeed, this approach has been successfully used in RHex for rough terrain [43] and stair climbing [44], etc. but only for very limited durations. In [45], a thermal observer is used to monitor core motor temperatures during the robot's operation. For a vertical climber, however, power requirements are large and roughly constant, reducing the relevance of intermittent operation. Nevertheless, by running the motors above continuously permissible currents, we are able to exceed the nominal power specification of the motor, if only for short periods of time. ${ }^{7}$

If the legs of the robot must only deliver maximum power during intermittent intervals (in this case, each leg's stance phase) and these intervals are phase offset from one another, a single, more powerful motor could be used to drive multiple mechanically coupled multiple joints or limbs. While the peak power draw would not decrease, a larger motor might be able to supply this power output without overheating. iSprawl [47], MechaRoach [48], and several toy robots have employed this approach. However, this imposes rigidly fixed leg trajectories which would not allow investigation of other behaviors and controllers, hindering the development of a more versatile climber.

3) Parallel Leg Springs: In order to maintain individual control of the legs and simultaneously overcome the power limitation of commercial motors, we utilize passive-elastic elements in parallel with the leg actuators. As suggested by the simplified numerical studies in [1], this method allows the legs to store energy during the swing-recirculation phase of their motion, while the power requirement is low, and then release the energy during stance to aid with accelerating the body upwards. In other words, we increase average mechanical power output without increasing the peak power demands on the motors themselves by providing means for the motors to do productive work throughout each stride instead of just during stance. Moreover, [46] demonstrates that motors operating continuously produce less thermal energy to a do given amount of mechanical work than those operating intermittently.

As reported in [1], the overall climbing speed of a onedimensional climber can be substantially increased by creating a second peak in the demanded power curve for each motor. Figure 6 shows a schematic of how the spring is used, and a plot of motor power as a function of time. The shaded areas represent the changes in the commanded torque with the addition of a spring in parallel with the actuator connecting each foot to the body.

The spring is at its unloaded, or rest, position when the foot is at its lowest position. As the foot extends in preparation for reattachment to the substrate, the spring is stretched, significantly increasing the torque required from the actuator. The maximum available motor torque, and the length of the crank linkage define the upper limit on the stiffness of this spring.

As shown in Fig. 6, with the addition of these legs springs $(\mathrm{k}=130 \mathrm{~N} / \mathrm{m}, \mathrm{b}=3 \mathrm{~N}-\mathrm{s} / \mathrm{m})$ the peak power required for each leg drops to just over $20 \mathrm{~W}$ during steady state climbing. Since the velocities of the cranks are unchanged, the locomotion dynamics of the system are the same as without the spring.

While this strategy solves the power problem during the steady-state climbing, a peak power output of more than $40 \mathrm{~W}$ is still required to accelerate the robot from rest. To better handle transient acceleration, a robot will require a more adaptive and efficient control scheme than the prescribed constant angular velocity scheme utilized for the animal template. A preliminary approach, described below, was utilized in the

${ }^{7}$ See [46] for a modern a motor sizing approach which explicitly considers thermal dynamics. 


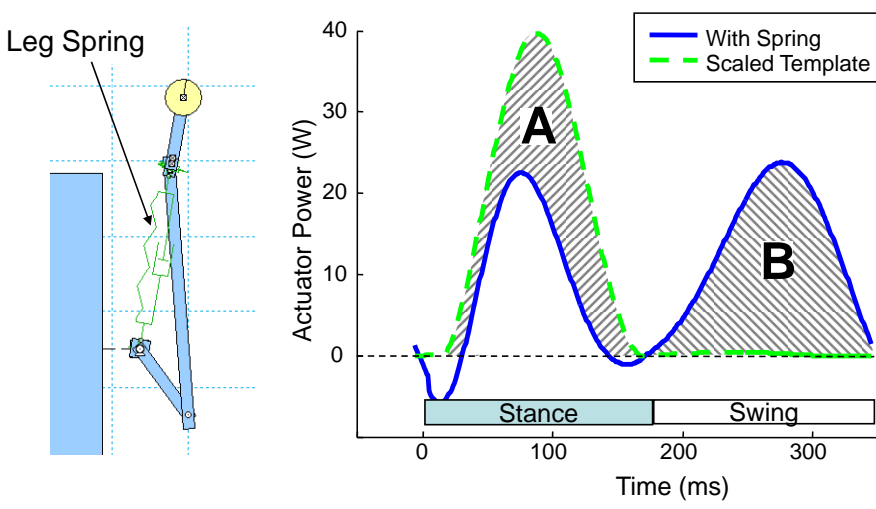

Fig. 6. Schematic of model with leg spring and the effect of the spring on the nominal torque profile. The 'A' region represents the effect of the spring assisting the motor, and the ' $\mathrm{B}$ ' region represents the work done by motor in stretching the spring.

design simulation, and a more refined version of this idea was implemented in the robot as described in Section V-B.

\section{Force Controlled Actuation}

To better exploit the robot's available on board power, we switch from commanding positions to commanding force - at least up to the actuators' abilities to deliver it. By explicitly regulating the motors' outputs rather than relying on position tracking errors, the actuators can be used to produce a greater amount of positive work during a stride. At the same time, this control framework sidesteps the need to design different reference motions for the vastly different operating regimes the robot encounters on its transient from standstill to steady state. Controlling them, instead, to extract the greatest possible amount of work against gravity regardless of operating regime enables the actuators to build up body center of mass speed over a number of strides, in an autonomous manner more naturally suited to the range of transient conditions encountered along the way.

In the first subsection below, we summarize our numerical feasibility studies [1] of a simple work-directed controller aimed at extracting the greatest possible amount of force from the actuators. The results from this study suggest that switching from a position to a force-based controller and adding leg springs can roughly double the robot's speed, and that in an ideal (no friction) case, climbing near $2 \mathrm{bl} / \mathrm{s}$ should be achievable for a $2 \mathrm{~kg}$ system.

Next, the work-directed control introduces a new design freedom that must also be addressed. The robot's two limbs must be properly coordinated so that one recirculates while the other is working — a simple matter in the setting of a reference position tracking control, but no longer intrinsically specified by the work-directed controller. We introduce a naive leg coordination controller scheme below in order proceed with the rough design study.

The implementation problem of designing a concrete workdirected scheme in the physical platform that can be guaranteed to stabilize these two different dynamics is taken up again in Section IV.
1) Motor model and controller: In order to maximize the output power of the motors without causing them to overheat, a model of their behavior must be incorporated into the control scheme. Figure 7 depicts the simple motor model used in our controller. The slope of the torque/speed curve depends on the particular motor chosen and on the gear ratios used in the power train.

We use the values provided by Maxon for our 20W DC motors [33]. The effective gear reduction $(G)$ was chosen to match the template dynamics and maximize speed.

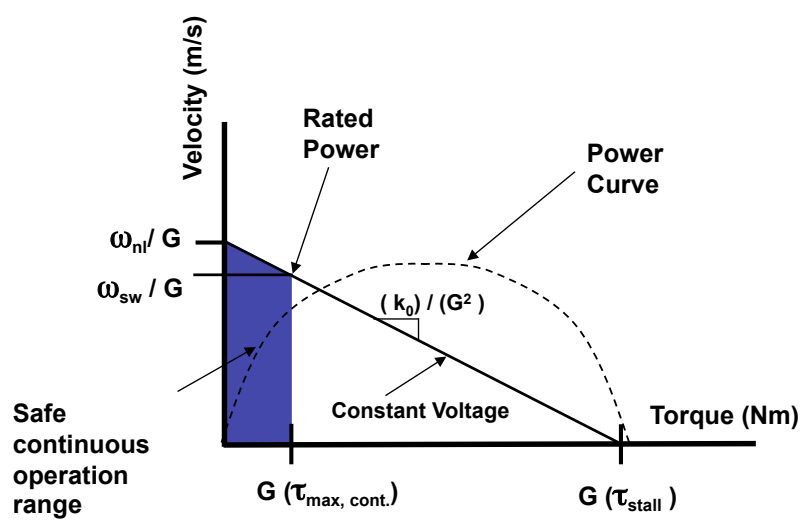

Fig. 7. Simplified geared motor model. The dashed power curve shows that maximum power output corresponds to loads equal to one half of stall.

Our (simulation) controller varies motor terminal voltage to ensure that the motor operates on the boundary of the shaded continuous operation zone shown in Fig. 7. At high speeds, the motor is supplied with the maximum (supply) voltage, while at lower speeds our controller restricts the voltage supplied to the motor so as not to exceed the motor's continuously permissible current rating. Thus, after applying our controller, the ouput torque is given by:

$$
\tau(\omega)=\min \left(\tau_{\max , c},\left(1-\frac{\omega}{\omega_{n l}}\right) G \cdot \tau_{\text {stall }}\right),
$$

where $\omega$ is the rotational speed of the motor shaft, $\tau_{\max , c}$ is the maximum continuous torque, $\omega_{n l}$ is the no-load speed, and $\tau_{\text {stall }}$ is the stall torque as specified by the motor manufacturer.

It should be noted that the introduction of this motor model provides a serious constraint on both the maximum power delivered by the motor and on the achievable torques, as our controller does not permit "thermal borrowing".

2) Leg coordination: A new issue arising from the introduction of the force-maximization controller is the challenge of ensuring the proper anti-phase coordination of legs. An earlier analytical study of coupled oscillatory climbing systems has shown that the limbs of these systems naturally phase lock, resulting in extremely large double support and aerial phases [49]. Our two-dimensional simulation exhibits the same tendency, with potentially catastrophic consequence for a physical climbing machine whose attachment mechanics are likely to preclude recovery from conditions of free-fall [50], 
[51]. Consequently, a mechanism is required to ensure proper phasing of the legs. We initially utilize the following braking heuristic algorithm:

$$
\operatorname{if}\left(\left(\theta_{i}<\epsilon\right) \text { and }\left(\theta_{j}<\pi\right)\right) \text { then } \tau_{i}=-c_{b} \tau_{\max }
$$

Where $\theta_{i}$ and $\theta_{j}$ represent the swing and stance legs, $\tau_{\max }$ is the maximum available torque from the hip motor, and $\epsilon$ is about $\pi / 10$ radians, and gait transitions occur when the leg phase angle $\theta=\pi$. By applying a negative torque to an ahead-of-schedule swing leg a fraction of a radian before touchdown, the controller attempts to ensure that touchdown occurs immediately after liftoff, just as in the template model.

The phase regulation just described lends itself to simple numerical implementation and affords the preliminary insight we require at this stage of design but it does not enjoy any analytical guarantees and is not parametrically well conceived (in contrast to the more careful scheme that we will introduce for actual implementation on the physical robot in Section VI): both the retarding factor, $c_{b}$, and the angular end-of-swing threshold, $\epsilon$ must be tuned to generate desirable behavior, lest a change in the actuator model or system dynamics result in extended duty factors or significant aerial phases. While the controller does, indeed, maximize power output to the stance leg, it is suboptimal in terms of efficiency and performance since it actively brakes just before stride transitions, rather than appropriately reducing the input energy throughout the swing phase. Based on these observations, a more advanced algorithm was developed for the robot, described here in Section V-B.

Despite its limitations, this algorithm effectively implements the leg coordination necessary to achieve repeatable climbing and, as we observe in the next section, this results in a simulation with performance that closely resembles that of the scaled version of the simple template.

3) Performance of simulated force controlled and phase regulated actuation schemes: Figure 8B shows the effects of switching from a position-based control to a max-force based control scheme on the performance of the simulated climber.
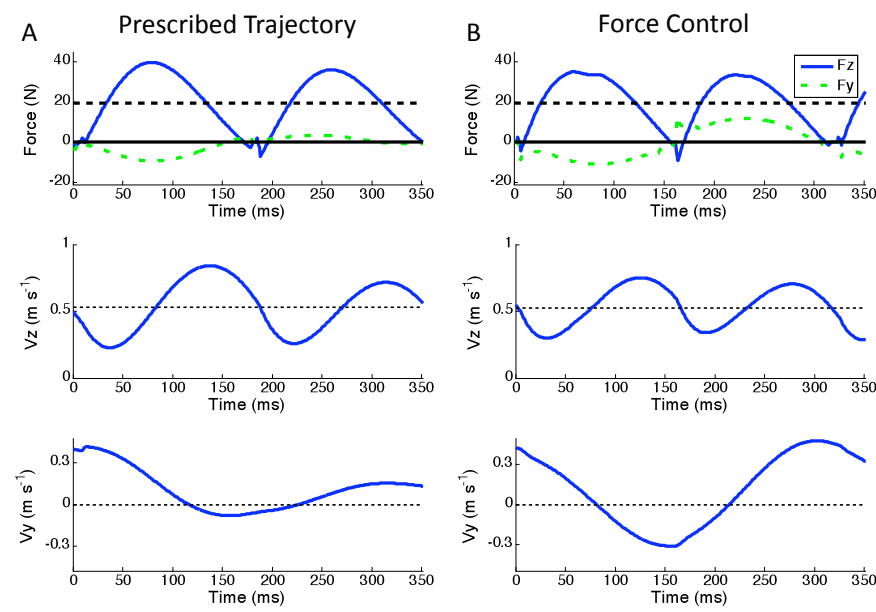

Fig. 8. Force, vertical velocity, and lateral velocity for a stride with (A) the trajectory-based and (B) force-based control schemes.
A comparison of the two models' dynamics reveals that, while there are subtle behavioral differences between the models, their behavior is largely equivalent.

The switch from a trajectory-tracking to a force-based control scheme results in a relinquishment of our control of the overall actuation frequency. While this frequency shifting during climbing can increase the performance of the robot, it also complicates the dynamic coupling between the leg switching, body rotation, and wrist-spring extension by introducing additional actuator dynamics. Concerns about potentially adverse dynamical interactions are addressed in the next section.

The net result is a realistically sized and powered dynamic climber that is very close to the template derived from animal studies. The robot's projected vertical speed of $0.55 \mathrm{~m} / \mathrm{s} \mathrm{com-}$ pares very favorably to that of the scaled template $(0.60 \mathrm{~m} / \mathrm{s})$.

\section{Simulation conclusions}

The addition of a force-assist spring in parallel with the actuator in the legs and the switch to a force-maximizing control scheme allow a simulated robot to climb dynamically at our target mass of $2 \mathrm{~kg}$.

According to (2), a climber's power-to-weight ratio must increase as the square root of length to preserve dynamic similarity. Thus, any increase in size requires an increase in power density. Since DynoClimber, when equipped with passiveelastic energy storage and an aggressive force-maximizing controller, is just able to achieve the power density required to climb dynamically, a substantial increase in size would mandate that the robot achieve an unrealizable power density. As shown in Section IV-B, attempting to climb when underpowered has a severe effect on upward speed. Thus it appears that with our present motor power density and control approach this anchor represents an approximate upper limit on speed at near-term utilitarian length scales for the FullGoldman template.

\section{Stability of the Internal Climber Dynamics}

Simulations presented in [19] indicate that the FullGoldman template converges to and climbs with a stable, period-1 gait. The template's prescribed-leg-length actuation scheme (described by (1)) rigidly dictates leg frequency and therefore makes asymmetrical or irregular gaits unlikely to appear. However, to anchor the template in a robot, the previous section has introduced two proposed changes to the template's actuation scheme. First, in Section IV-B, we introduce parallel energy-storage springs to reduce the (peak) power density required for dynamic climbing, and second, in Section IV-C, we equip the proposed mechanism with a control policy that permits us to maximize actuator power output (losing precise specification of leg frequency).

By design, our controller causes the climber's motors to operate along their speed-torque curve, and therefore exposes it to the motors' dynamics, speed/force trade-off, and inherent power limitations; the parallel spring introduces yet another dynamical exchange of potential and kinetic energy. The addition of these dynamics raises a concern that the coupled electromechanical plant may exhibit unstable or unfavorable 
oscillations which do not appear in the template. While our design simulation remarkably appears to climb without spurious effects caused by these modifications, through simulation alone we are unable to comprehensively assess the robustness of the climber's behavior to parameter or initial condition variations.

In this section we formalize the foregoing design models, simplifying and decoupling them to the extent that mathematical guarantees can be established precluding the possibility of such undesired dynamical instabilities over any initial conditions for any physically reasonable choice of design parameters. We first consider the vertical power stroke in isolation from the leg alternation dynamics and give a proof that a simplified one degree of freedom abstraction of the resulting dynamical system is globally asymptotically stable. We next introduce a more parametrically parsimonious (yet still analytically tractable) phase regulation controller and give a proof that our phase alternation dynamics, in isolation from the mechanical power stroke dynamics is also globally asymptotically stable.

\section{A. Power stroke modeling and analysis}

The Full-Goldman template's counterparts for level ground running - the SLIP and LLS templates - have generated a more-than-decade long literature [52] and remain an active area of research in legged locomotion. Early analysis [53] of the first dynamical running machines [54] demonstrated that a poorly tuned actuation subsystem could be responsible for the period two (or higher) "limping gaits" observed in vertical hopping [53] and juggling [55]. Even the simplest two degree of freedom model of the unactuated SLIP templates displays higher order (period-2) limping gaits in a physically relevant parameter regime [56]. It is notable, then, that the design simulation does not seem to exhibit period-2 gaits. Thus, two factors contribute to our motivation to undertake at least a rudimentary analysis of our climber's stability: our introduction of actuator dynamics, and the precedence of higher-period gaits in prior locomotion templates.

In the tradition of that past literature we introduce in this first analysis of the DynoClimber design-prototoype the simplest model that can still capture the crucial interaction of mass and motor dynamics: a one degree of freedom representation of our climber's "power train" — the dynamics of force-actuated vertical climbing, independent of rotational dynamics. ${ }^{8}$ To that end, we build a simplified mathematical model composed of a point mass propelled vertically upward by an actuator.

When instantiated with a range of physically motivated parameter values that includes those characterizing our particular robot prototypes, this vertical power stroke model converges to a period-1 gait from all initial conditions as we will now show. While not a conclusive statement as to the stability of the physical coupled system, this result demonstrates that the

\footnotetext{
${ }^{8}$ In contrast, as for all multi-jointed locomotion models [53, 54, 56], the complete template (Fig. 1) entails non-integrable dynamics, whose mathematical analysis will likely require as similarly long a string of focused papers as occasioned by level ground running $[52,53,54,56]$.
}

power stroke dynamics cannot in and of themselves be a source of instability or parasitic, higher period dynamics.

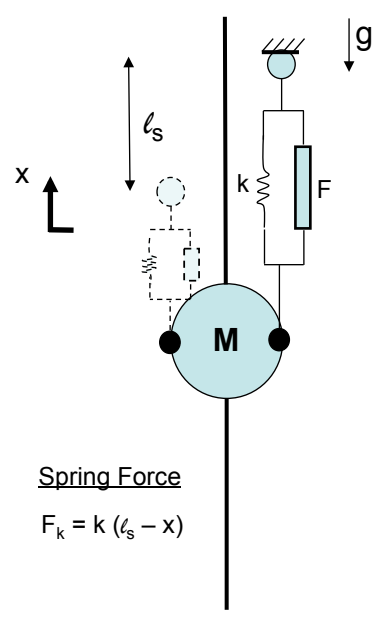

Fig. 9. Schematic of the climbing model used. The spring is fully loaded when the leg reaches full extension.

1) Vertical power stroke model : Depicted in Fig. 9, the vertical power stroke model consists of a rigid body of mass $M$ and two massless legs operating in a one dimensional workspace - the climbing "wire" - subject at all times to a constant gravitational force $M \cdot g$. By construction, exactly one foot is attached, and applying force, to the substrate at all times. Since the feet are massless, the unattached foot does not affect the climber's upward progress.

A foot begins its stance phase by attaching to a fixed point on the substrate with its corresponding leg extended to the full stride length, $l_{s}$. That leg applies a contractile force (described in detail in the next paragraph) propelling the climber's mass upward. When the mass has traveled a vertical distance upward of $l_{s}$, the foot in contact with the wall instantaneously breaks contact, while the other foot simultaneously gains contact, once again at a distance of $l_{s}$ from the mass.

The stance-phase contractile force consists of two elements intended to resemble the actuator used in ours design simulation: an energy-storage spring with Hooke Law constant $k$ in parallel with a motor-based force $F$. The energy storage spring begins each stride producing maximal upward force, $l_{s} \cdot k$. That force diminishes throughout the stride until the stride terminates with the spring at its rest length, producing a force of 0 . The motor-based force is given by a motor and gearbox driving a simulated rack and pinion transmission mechanism. We approximate the physical transmission - a crank-slider by an ideal rack and pinion to promote analytical tractability.

The motor produces a rotational torque which is amplified linearly by the gearbox with gear ratio $G$. This force drives the simulated pinion (with radius $r$ ) which proportionately converts the torque from the gearbox to a linear force. Thus, a motor torque $\tau_{m}$ produces a prismatic output force of $F=\frac{G}{r} \cdot \tau_{m}$.

The control scheme is as simple as possible: the stance leg's motor is assigned a constant voltage at all times. We demonstrate in Section VI-A that this scheme results in 
identical steady-state behavior to the controller in Section IV$\mathrm{C}$, but requires less electrical and software infrastructure to implement. At a speed of 0 , the motor produces its full stall torque of $\tau_{s}$, and motor torque production decreases linearly with motor speed until the motor produces a torque of 0 at its no-load speed $\omega_{n l}$, as per the standard linear motor model [33, 57]. It bears noting that $\tau_{s}$ and $\omega_{n l}$ are given for a nominal voltage; changing the voltage applied to the motor scales each of these terms linearly.

The parameters used to define the vertical power stroke model are summarized in Table IV. Note that throughout this section, we rarely refer to torques and angular speeds, preferring the equivalent linear forces and velocities.

Figure 10 introduces the coordinates $z=\left(z_{1}, z_{2}\right)=(z, \dot{z}) \in$ $\mathcal{Z}=: \mathbb{R}^{2}$ and depicts the physically relevant state space of the climbing model. This is a rectangle in the upper half plane bounded by the abscissa below, $\tilde{\mathcal{L}}_{2}$, indicating an upward velocity of 0 , and two vertical lines, the demarcation of kinematic limits. The ordinate to the left, $\mathcal{L}_{1}$, at $x=0$, and a parallel line, $\tilde{\mathcal{L}}_{1}$, to the right, at $x=l_{s}$ bound the physically permitted stroke length and extension of the spring. When the system trajectory intersects $\tilde{\mathcal{L}}_{1}$, the reset function $s$ is applied and a new stride begins with the system trajectory lying along $\mathcal{L}_{1}$ with the same coordinate value as before. This reset map, $s$, is depicted in Fig. 10: it corresponds to the termination of one leg's stance phase and simultaneous attachment of the other leg to the wall.

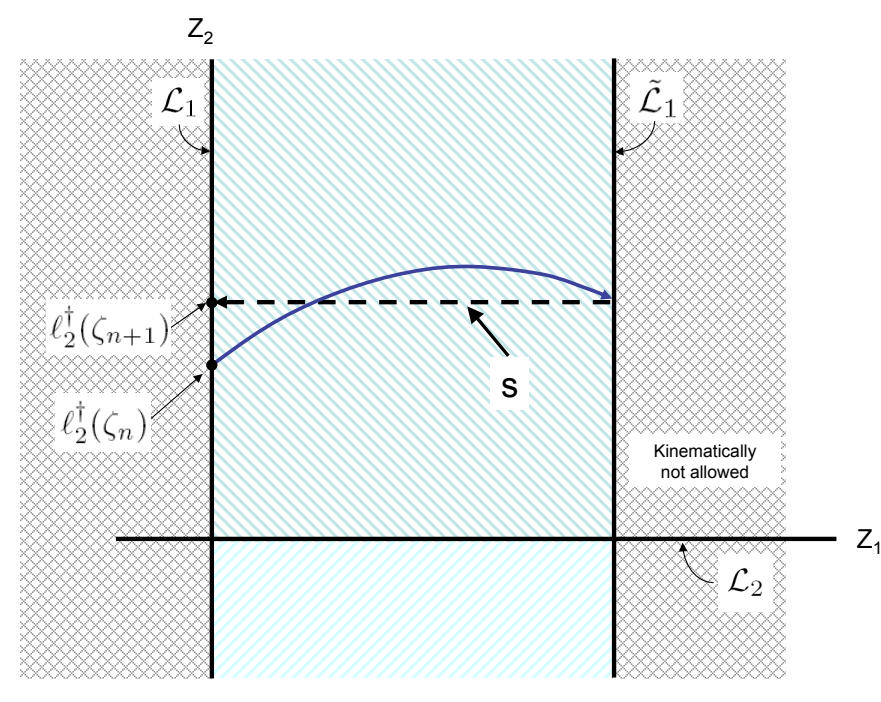

Fig. 10. Patches, boundary sets, and sample trajectory from initial condition $\zeta_{n}$ on $\mathcal{L}_{1}$ to $\tilde{\mathcal{L}}_{1}$, then projected back onto $\mathcal{L}_{1}$, via the reset function $s$, to represent the next iterate under the return map, $\zeta_{n+1}$ for the simplified climber.

Using $z \in \mathcal{Z}$ coordinates, the equations of motion for the body, with mass $M$, can be written by inspection from Fig. 9 as:

$$
\begin{gathered}
\dot{z_{1}}=z_{2} \\
M \dot{z_{2}}=F_{k}+F_{m}-M g
\end{gathered}
$$

where the spring force is $F_{k}=: k\left(l_{s}-z_{1}\right)$, and the motor force is

$$
F_{m}=: F_{s} \cdot\left(1-\frac{z_{2}}{v_{n l}}\right) .
$$

The resulting second order dynamics are given by:

$$
\dot{z}=A z+b
$$

with

$$
A=\left[\begin{array}{cc}
0 & 1 \\
-\alpha^{2} & -2 \cdot \sigma \cdot \alpha
\end{array}\right], b=\left[\begin{array}{l}
0 \\
\beta
\end{array}\right],
$$

where the terms used in (5) are summarized in Table I.

TABLE I

ELEMENTS OF THE EQUATIONS OF MOTION

\begin{tabular}{|c|c|l|}
\hline Term & Definition & Physical Meaning \\
\hline$\alpha$ & $\sqrt{\frac{k}{M}}$ & natural spring-mass frequency \\
$\sigma$ & $\frac{F_{s}}{2 \cdot M \cdot v_{n l} \cdot \alpha}$ & $\begin{array}{l}\text { Effective damping due to motor } \\
\text { back-EMF } \\
\text { net vertical force at stall }\end{array}$ \\
$\beta$ & $\frac{1}{M}\left(-M \cdot g+k \cdot l_{s}+F_{s}\right)$ & netical \\
\hline
\end{tabular}

2) Return map: As is standard, our analysis focuses on the discrete time iterates of a return map [58]. In this case, the velocity of the climbing mass is sampled at an event (the "section") which is revisited again and again as the robot climbs. The section employed here is the touchdown of the next extended limb; this occurs when a system trajectory intersects $\tilde{\mathcal{L}}_{1}$ and the reset function $s$ is applied. By examining the velocity of the climber at the beginning of each stride, we demonstrate analytically that it converges to a period-1 gait.

We will write the return map, $R$, using section coordinates $\zeta \in \mathcal{L}_{1}$, as depicted in Fig. 10. This scalar variable, $\zeta$, represents physically the climber's vertical velocity at the start of a stride. Trajectories arise as the composition of the flow from $\mathcal{L}_{1}$ to $\tilde{\mathcal{L}}_{1}$ with a "swing resetting event" $(s)$ that maps the body state associated with a completely compressed arm at a given velocity in $\tilde{\mathcal{L}}_{1}$ to the same body state associated with a completely extended (contralateral) arm at the same velocity in $\mathcal{L}_{1}$. The reset function $s$ that formalizes this swing resetting event can now be written down directly as:

$$
s\left(z_{1}, z_{2}\right):=\left(0, z_{2}\right)
$$

We denote by the symbol $f^{t}(z)$, the time trajectory of the dynamics (5) through some initial condition, $z$. We also define the projection operators $\ell_{1}\left(z_{1}, z_{2}\right):=z_{1}$ and $\ell_{2}\left(z_{1}, z_{2}\right):=$ $z_{2}$, as well as the pseudo-inverse $\ell_{2}^{\dagger}\left(z_{2}\right):=\left(0, z_{2}\right)$ Because we are interested in initial conditions on the section, $\mathcal{L}_{1}$, we parameterize these trajectories by $\zeta \in \mathbb{R}$ via composition with $\ell_{2}^{\dagger}$ and $\ell_{2}$. Using these functions, we define the return map, $R:=\ell_{2} \circ s \circ f^{T} \circ \ell_{2}^{\dagger}(\zeta)$, where $T$ is the time taken to the end of the stride.

$R$ quantifies how the system evolves from the beginning of one step to the beginning of the next. It is comprised of the flow induced by the vector field (5), and the reset function $s$. Details of the derivation of the return map can be found in the Appendix. The return map can be expressed as: 


$$
\begin{gathered}
R(\zeta)=\frac{1}{2 \alpha \sqrt{\sigma^{2}-1}} \cdot \\
\left(\lambda_{1} e^{\alpha \lambda_{1} T}\left[\beta \lambda_{2}+\alpha \zeta\right]-\lambda_{2} e^{\alpha \lambda_{2} T}\left[\beta \lambda_{1}+\alpha \zeta\right]\right)
\end{gathered}
$$

Note that $T$ is defined implicitly as a function of $\zeta$ (see the Appendix for a careful definition), making a closed form solution of the return map impossible. However, we are able to investigate the stability properties of the return map by examining its derivative, $D R$, derived in the appendix, and written in more compact form as

$$
D R(\zeta)=\frac{(2 \rho d)\left(e^{-\alpha \sigma T}\right)}{\rho\left(d^{2}+1\right)+c\left(d^{2}-1\right)},
$$

where for ease of analysis we introduce the following substitutions:

$$
\begin{aligned}
\rho(\zeta) & :=\zeta \alpha \sqrt{\sigma^{2}-1} \\
c(\zeta) & :=\beta-\alpha \sigma \zeta \\
d(\zeta) & :=e^{T a(\zeta) / \zeta} .
\end{aligned}
$$

3) Physically imposed flow boundaries : To analyze the properties of the flow precisely we examine the acceleration null-cline (zeros of the acceleration component of the vector fields) located on the affine set:

$$
\mathcal{N}:=\left\{\left(z_{1}, z_{2}\right) \in \mathbb{R}^{2} \mid z_{2}=\frac{\beta}{2 \sigma \alpha}-z_{1} \cdot \frac{\alpha}{2 \sigma}\right\}
$$

This defines a region below $\mathcal{N}$, as depicted by the " + " sign in Fig. 11, within which the trajectory experiences an increase in vertical velocity due to the accelerating effects of the actuator and spring. We will find it useful to impose a partition of the section $\mathcal{L}_{1}$ by labeling the special point where it intersects the nullcline,

$$
\zeta_{B}:=\ell_{2}\left(\mathcal{N} \cap \mathcal{L}_{1}\right)
$$

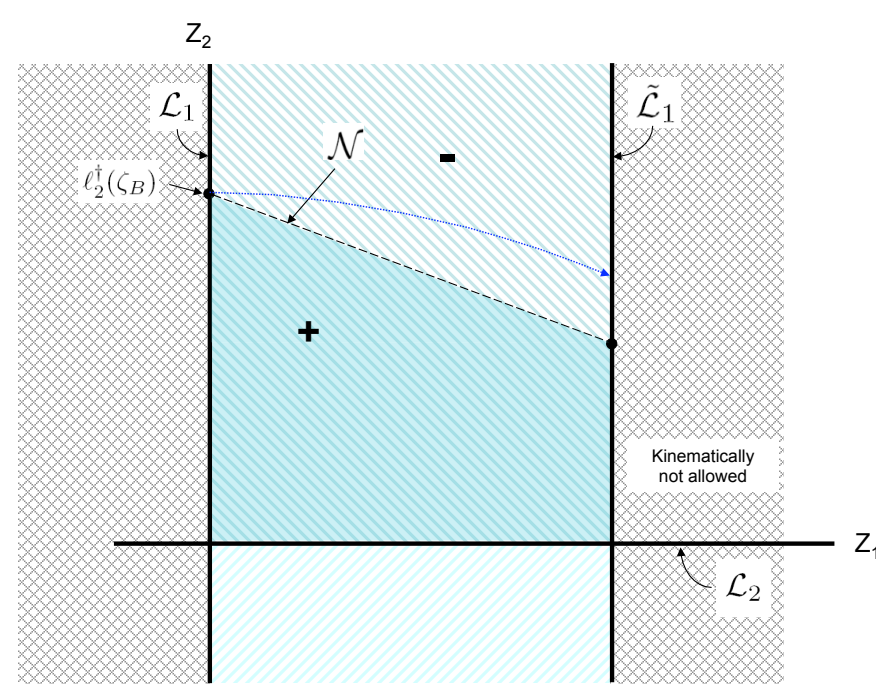

Fig. 11. The null cline of the system $(\mathcal{N})$ overlaid on the patches and boundary sets of the sytem. For states above and to the right of the null clines the vector field is increasing (indicated by the '+ 'signs), and beneath and to the left the vector field is decreasing (indicated by '- 'signs).
Beginning with this initial condition, a climber's spring and motor together provide just enough force to support its mass at the beginning of the stride. In the proof that follows we observe that trajectories initiating with this and all larger velocities remain above the nullcline and hence experience decreasing vertical velocity throughout the stride.

4) Proof of stability: We now demonstrate that the climber's return map has a globally asymptotically stable fixed point, meaning that from every initial condition the climber converges to a stable period- 1 gait. The proof entails establishing 3 claims:

a) The return map $R$ has a slope between 0 and 1 over interval $I=\left[0, \zeta_{B}\right]$.

b) $I$ is invariant under $R$, Additionally, $I$ is attractive from above.

c) Starting from any initial velocity $\zeta_{0} \geq 0$, iterates of the return map converge to a unique fixed point:

$\lim _{n \rightarrow \infty} R^{n}\left(\zeta_{0}\right)=\zeta^{*}$

We make several assumptions about system parameters throughout this section. These algebraic assumptions, listed in Table II, comprise a sufficient, but not necessary, set of conditions for our stability result, and they are clearly satisfied for any reasonable physical design (including all of our prototypes) as shown in the table.

TABLE II

PARAMETER ASSUMPTIONS APPLIED TO MODELS FROM TABLE IV

\begin{tabular}{|c|c|c|c|}
\hline Quantity & Assumption & Initial Model & Revised Model \\
\hline$\beta$ & $>0$ & 161 & 197 \\
$\sigma$ & $>1$ & 27.5 & 16.6 \\
$\alpha$ & $>0$ & 4.92 & 4.72 \\
\hline
\end{tabular}

The third assumption requires special note. The condition that $\alpha>0$ is equivalent to $k>0$ : we assume that our climbing model employs an energy storage spring. This assumption is not required for stability, but, rather, is critical to establish the mathematical correspondence to a salient aspect of Dynoclimber's power train design as detailed Section IV. ${ }^{9}$

In contrast, $\beta$ gives the net force on the climber as it begins a stride with no upward velocity. Should $\beta$ not be larger than 0 , the climber would not move upward. Moreover, since the motor's torque production decreases monotonically with climber velocity, a climber with $\beta \leq 0$ would always operate above the null-cline in state space; in essence, it would be unable to climb.

Finally, $\sigma>1$ is established as a convenient sufficient condition to simplify the proof and is handily achieved by our platforms (Table II). Specifically, $\sigma>1$ assures that both eigenvalues of the system are real and negative (see appendix for exact expression), with $\lambda_{1}>\lambda_{2}$. From the definition of $\sigma$, it is evident that $\sigma>1$ for any climber reasonably similar to DynoClimber. Only an extremely under-geared climber could

${ }^{9}$ Were $k=0$, the analysis would be drastically simplified, as the climber's hybrid dynamics would become trivial. The vector field representing climber acceleration on $z_{1}, z_{2}$ would no longer depend on $z_{1}$, and the climber would simply converge to that constant velocity at which its motor produced a force of $M g$. Thus, while $k=0$ is a physically valid scenario, it is trivially stable and we do not emphasize its analysis. 
cause $\sigma$ to fall below 1; DynoClimber would have to employ a gear ratio of roughly 14:1 instead of the nearly 57:1 gearing used in the most recent version of the robot.

Proposition 1: Defining $I=\left[0, \zeta_{B}\right]$, The return map $R$ has a bounded slope over this interval: $\forall \zeta \in I, D R(\zeta) \in(0,1)$.

Proof: First we show that $R$ is strictly monotonic: $D R(\zeta)>0$. To that end, we examine $D R$ from (7),

$$
D R(\zeta)=\frac{(2 \rho)\left(e^{-\alpha \sigma T}\right)(d)}{\rho\left(d^{2}+1\right)+c\left(d^{2}-1\right)},
$$

and demonstrate that its numerator and denominator have the same sign (in this case, positive).

We note that $\rho>0$, since $\zeta>0$ (the climber begins with a positive velocity), $\alpha>0$ (there is an energy storage spring), and $\sigma>0$ (stall torque and no-load speed are positive).

Since $d>1$ by inspection, each term in this expression is positive if $c(\zeta)=\beta-\alpha \sigma \zeta>0$. Noting that $c(\zeta)$ is strictly decreasing in $\zeta$, it is evidently minimized over $\zeta \in I$ when $\zeta=\zeta_{B}$. Expanding $M \cdot c(\zeta)$ into physical parameter values and simplifying,

$$
\begin{aligned}
M \cdot c(\zeta) & =\quad\left(-M g+k l_{s}+F_{s}\right)-\frac{F_{s}}{2} \cdot \frac{\zeta}{v_{n l}} \\
& =\quad-M g+k l_{s}+F_{m}(\zeta)+\frac{F_{s}}{2} \cdot \frac{\zeta}{v_{n l}}
\end{aligned}
$$

where $F_{m}$ is the vertical force produced by the motor as a function of velocity. To evaluate $c$ at $\zeta=\zeta_{B}$, we recall the meaning of $\zeta_{B}$ : this is the velocity at which the climber has a net acceleration of 0 at the beginning of a stride. Thus, $F_{m}\left(\zeta_{B}\right)=M g-k l_{s}$, implying that

$$
c\left(\zeta_{B}\right)=\frac{F_{s}}{2} \cdot \frac{\zeta_{B}}{M \cdot v_{n l}}>0 .
$$

Since $c(\zeta)>0$ over $I, \forall \zeta \in I, D R(\zeta)>0$.

Next, we demonstrate that $D R<1$

We aim to show that the numerator of $D R(\zeta)$ is smaller than its denominator. Subtracting the numerator of (7) from the denominator and simplifying:

$$
\begin{aligned}
& \rho\left(d^{2}+1\right)+c\left(d^{2}-1\right)-(2 \rho)\left(e^{-\alpha \sigma T}\right)(d) \\
& >\quad \rho\left(d^{2}+1\right)+c\left(d^{2}-1\right)-2 \rho d= \\
& =\quad \rho(d-1)^{2}+c\left(d^{2}-1\right)>0
\end{aligned}
$$

Thus, $D R(\zeta)<1$ for all $\zeta \in I$.

Proposition 2: $\forall \zeta \in I, R(\zeta) \in I$.

Proof: Since Prop. 1 establishes that $R$ is monotonically increasing with slope less than 1 over $I$, we need only demonstrate that $R(0) \geq 0$ and that $R\left(\zeta_{B}\right) \leq \zeta_{B}$ to establish the invariance of $I$. Using the return map derived in (6) and noting that $\lambda_{1} \cdot \lambda_{2}=1$,

$$
R(0)=\frac{\beta}{2 \alpha \sqrt{\sigma^{2}-1}} \cdot\left(e^{\alpha \lambda_{1} T}-e^{\alpha \lambda_{2} T}\right)
$$

Since $\lambda_{1}>\lambda_{2}$ and all other constants are positive, $R(0)>0$ for any $T>0$.

Looking at the flow of the system beginning at $\zeta_{B}=$ : $\ell_{2}\left(\mathcal{N} \cap \mathcal{L}_{1}\right)=\frac{v_{n l}\left(F_{s}+k l_{s}-g M\right)}{F s}$, we show that the system's trajectory remains above $\mathcal{N}$ on the $\left(z_{1}, z_{2}\right)$ plane by looking at the vertical distance between the two:

$$
\begin{aligned}
\ell_{2} \circ f^{t} \circ \ell_{2}^{\dagger}\left(\zeta_{B}\right)-\left(\frac{\beta}{2 \sigma \alpha}-\ell_{1} \circ f^{t} \circ \ell_{2}^{\dagger}\left(\zeta_{B}\right) \cdot \frac{\alpha}{2 \sigma}\right) & = \\
\frac{\left(e^{t \alpha \lambda_{1}}-e^{t \alpha \lambda_{2}}\right) \beta}{8 \alpha \sigma^{2} \sqrt{\sigma^{2}-1}} & \geq 0 .
\end{aligned}
$$

Again, since $\lambda_{1}>\lambda_{2}$, the expression above is positive. This demonstrates that the climber's velocity stays above the null cline $\mathcal{N}$ throughout the stride, implying that the climber's velocity is strictly decreasing. Thus, $R\left(\zeta_{B}\right)<\zeta_{B}$.

Corollary 1: $I$ is attractive from above.

Proof: $\forall \zeta>\zeta_{B}, f^{t} \circ \ell_{2}^{\dagger}(\zeta)$ lies above $\mathcal{N}$ on the $\left(z_{1}, z_{2}\right)$ plane. Thus, for any initial condition $\zeta>\zeta_{B}, R^{n}(\zeta)<\zeta_{B}$ for sufficiently large $n$.

Proposition 3: For all $\zeta_{0} \geq 0$, iterates of the return map $R^{n}\left(\zeta_{0}\right)$ converge to a fixed point. $\zeta^{*}$, as $n \rightarrow \infty$.

Proof: We proceed by demonstrating that $R$ is a strict contraction in $I$ and applying the Contraction Mapping Principle. Thus, we first demonstrate that $\forall \zeta_{1}, \zeta_{2} \geq 0$, and $\zeta_{1} \neq \zeta_{2}$, $\left|R\left(\zeta_{2}\right)-R\left(\zeta_{1}\right)\right| \leq k \cdot\left|\zeta_{2}-\zeta_{1}\right|$, where $k \in(0,1)$

$\left|R\left(\zeta_{2}\right)-R\left(\zeta_{1}\right)\right|=\left|\int_{\zeta_{1}}^{\zeta_{2}} D R(\zeta) d \zeta\right|$. Since $D R<1$ by Prop. 1 , and $D R$ is continuous on $I, D R$ must never exceed some $k<1$ on the interval $I$. Thus, $\left|\int_{\zeta_{1}}^{\zeta_{2}} D R(\zeta) d \zeta\right| \leq\left|\int_{\zeta_{1}}^{\zeta_{2}} k d \zeta\right|=$ $k\left|\zeta_{2}-\zeta_{1}\right|$.

Since $R$ is a strict contraction on a complete space $I$, it has a unique fixed point with $I$ as its basin of attraction by the Contraction Mapping Principle. Moreover, since $I$ is attractive from above, the fixed point of $R$ has a basin of attraction of $\overline{\mathbb{R}^{+}}$.

5) Summary discussion of the power stroke model analysis: Our power stroke model — if equipped with parameters within the physically relevant range introduced in Table II - converges to a steady-state, non-limping gait, represented by the return map fixed point, $\zeta^{*}$ determined in Prop. 3 and representative of the physical average climbing velocity

$$
\bar{\zeta}^{*}:=\frac{1}{T} \int_{0}^{T} \ell_{2} \circ f^{t} \circ \tilde{\ell}_{2}\left(\zeta^{*}\right) d t .
$$

Thus, the introduction of an actuator-model-equipped power stroke does not itself introduce instability.

This analysis of a simplified one-dimensional model has utility in terms of understanding the underlying dynamics of climbing, and in informing future design decisions. It has shown that for parameter ranges that correspond to robot designs of interest the dynamics of this hybrid system in the vertical direction are naturally self-stabilizing. This global stability behavior inheres notwithstanding the absence of mechanical damping. Instead, motor back-emf acts as a damper, monotonically reducing motor force as a function of the climber's vertical speed.

In ongoing work, we are investigating the stability properties of a more general class of climbing dynamics models. For purposes of the present paper, however, this analysis supports the conclusion that the use of passive energy storage elements to distribute the load on the actuator over a wider percentage of the cycle results in faster climbing without adversely affecting 
the vertical stability of our particular climber. With confidence in the underlying dynamic properties we can proceed with the construction and physical testing of the physical power train.

\section{B. Coordination controllers}

The two physically implemented versions of DynoClimber use two distinct controllers, both of which are described here. Throughout, we refer to a leg attached to the wall as being in stance mode, and a leg which is detached from the wall as being in flight mode. All control code is run on a Linux-based $366 \mathrm{MHz}$ CPU card and custom designed carrier and motor control boards.

1) Mirror-law: Although the force controlled actuation scheme described in Section IV-C functioned adequately in simulation, concerns about the inefficiency of the "retarding factor" motivated the development of two increasingly-more refined force-based controllers to implement on the physical robot.

The first improvement upon that preliminary architecture is a 4-state hybrid controller designed to achieve a nearlyconstant $180^{\circ}$ phase difference between legs while still maximizing the power driving the stance leg at all times. The state of the controller is established by the stance/flight status of each of the legs.

Imposing the necessary anti-phase influences on feedbackdriven hybrid oscillators by recourse to a "mirror law" first introduced in [59], an approach we adopted with the first physical version of this robot. In this scheme, a leg in stance mode is commanded the highest permissible voltage, $V_{\text {stance }}=V_{\max }$, while the leg in flight mode is controlled to follow the stance leg with an offset of $\pi$, namely,

$$
V_{f l i g h t}=k_{p} *\left(\theta_{f}-\theta_{s}-\frac{\pi}{2}-\pi\right)+k_{d} *\left(\dot{\theta}_{f}-\dot{\theta}_{s}\right)
$$

where subtraction on the circle is done modulo $2 \pi ; k_{p}$ and $k_{d}$ are controller gains and $\theta_{f}$ is the position of the leg in flight mode, and $\theta_{s}$ in stance.

Although this controller improves upon the predecessor used in the design simulation, systematic difficulties remain. Most notably, a leg in flight mode uses error-based tracking to stay $180^{\circ}$ out of phase with the continually advancing stance leg; thus, the flight leg lags the stance leg during every stride, consistently threatening to impose a short but highly deleterious double-flight phase. In such cases, the legs are brought into the desirable stance-flight configuration by retarding the erstwhile stance leg in the beginning of its flight phase, while the other leg continues forward into stance as rapidly as possible. This control law results in empirical convergence to a limit cycle unfortunately it also abruptly slows each leg once every stride, albeit less than does its simulated predecessor. The abruptness in behavior is a consequence of the controller's intrinsic nonsmoothness originating in the hybrid transition between stance and flight mode.

In addition to slowing the robot's pace overall, the abrupt transitions required by the mirror-law controller result in substantial mechanical stress and electrical noise. Thus, complementing the robot's mechanical and electrical redesign (described in Section VI-A), we designed a controller which achieves the same goals as the mirror-law without such abrupt transitions.

2) Self-exciting, work-directed: With the goal of eliminating nonsmooth controller transitions and ultimately improving climbing behavior, while achieving a greater degree of mathematical tractability, a new controller was introduced concomitant with the modified physical platform.

This controller, like both its predecessors, employs a selfexciting (clock-free) approach which, in contrast to its mirrorlaw predecessor, admits an analytical proof of stability, while obviating any need for gain tuning to ensure tracking (this proof is demonstrated in the next subsection, Section V-B.3). The control law is written in terms of the commanded voltage signal $V=:\left(V_{1}, V_{2}\right)$ and the difference between the motor shaft angles $\Theta=\left(\theta_{1}, \theta_{2}\right), \delta=\theta_{1}-\theta_{2}$ where,

$$
V(\Theta)=V_{\text {Max }}\left[\begin{array}{l}
1 \\
1
\end{array}\right]-k_{r} \sin ^{2}(\delta) \cdot\left[\begin{array}{c}
\mathrm{u} \circ \sin (-\delta) \\
\mathrm{u} \circ \sin (\delta)
\end{array}\right]
$$

where $V_{\text {Max }}$ is the maximum voltage and the unit step function, $u$, outputs the scalar value 1 if its argument is positive and outputs 0 elsewhere.

The retarding gain, $k_{r}$, determines the transient behavior of the system; a larger $k_{r}$ forces the system to converge more quickly, at the expense of speed of oscillation during the transient period and any time the system is perturbed from its limit cycle. With a $k_{r}$ near 0 , on the other hand, the system will return more slowly to its limit cycle during any transient period, but both motors will, on average, be commanded higher voltages while the system is away from its limit behavior. As shown in the next section, regardless of the choice of $k_{r}$, the system provably converges to a limit cycle with a velocity which does not depend on the retarding gain. Moreover, as long as $k_{r}$ is kept between 0 and 2, the controller will not exceed the specified maximum voltage, $V_{\text {Max }}$. For the experiments described in Section VI-D, $k_{r}$ has been fixed equal to 0.5 . This controller effectively implements hybrid transitions smoothly and in a provably correct way.

As shown in Section VI-D, this controller permits the robot to climb at an unprecedented pace and, anecdotally, substantially reduces the stress experienced by the robot's electrical and mechanical components.

3) Coordination dynamics: Given the empirical success of the self-exciting, work-directed controller, we now analyze its stability to be sure that it will accomplish the prescribed goals regardless of initial condition or parameter choices. Thus, in this section we prove the correctness of a class of self-exciting, work-directed controllers. One controller from that class was used for DynoClimber's most rapid climbing, and is presented in Section V-B.2.

We first introduce a simplified leg-coordination model of the physical motor system in order to provide an analytical basis for the success of our controller. This model is not intended to be accurate to our specific robot (as are, for instance, the design and power stroke models). Instead, we construct a general actuator model and prove that our controller functions as desired if applied to any actuator chosen from that class 
(including, of course, the specific actuator implemented on our platform).

Reflecting the morphology of DynoClimber, the legcoordination model consists of two identical motors with shaft angles $\Theta=\left(\theta_{1}, \theta_{2}\right) \in \mathbb{T}^{2}$, each subject to the traditional second-order linear motor model:

$$
\frac{\ddot{\theta}_{i} J R}{k_{\tau}}+\frac{\dot{\theta}_{i}}{k_{v}}=V,
$$

where $\theta$ is output shaft angle, $J$ is the moment of inertia of the commutator, output shaft and mechanism, $R$ is the winding resistance of the motor, $k_{\tau}$ is the torque constant of the motor, $k_{v}$ is the speed constant of the motor, and $V$ is the terminal voltage. This is an equivalent model to that employed in [60], with the caveat that we base our analysis on a system which supplies voltage, not current, to the motors.

It is important to note here that variable loading and frictional effects from the dynamics of climbing (manifested as substantial time variations in $J$ and $k_{v}$ ) dominate the behavior of the system, and any forces applied to the foot are reflected through a highly backdriveable mechanism as torques applied to the motor.

We thus wish to minimize our dependence on an accurate system model as the parameters of this model could vary widely based on the operating regime of the robot, and therefore generalize the motor model from (18) to include all constant inertia, Rayleigh-damped, Hooke's Law spring potential mechanical systems of the form

$$
k_{2} \ddot{\theta}+k_{1} \dot{\theta}=V
$$

where $k_{1}, k_{2}>0$. We construct a controller which will achieve its goals regardless of the choice of $k_{1}$ and $k_{2}$.

We represent the "robot" with two identical but independent actuator models, each standing in for one of the robot's motors and linkages:

$$
k_{2} \ddot{\Theta}+k_{1} \dot{\Theta}=\left[\begin{array}{l}
V_{1}(\Theta) \\
V_{2}(\Theta)
\end{array}\right]
$$

The controller is designed to dynamically "couple" these putatively independent motors through a memoryless nonlinear output feedback law that respects their terminal voltage magnitude constraints and guarantees that in the absence of external perturbations they will converge as a coupled system to the desired limit cycle on the torus of paired shaft angles and its tangent space of paired velocities from almost every initial condition. In employing this abstraction we explicitly neglect the motors' mechanical coupling through the body, and relegate the actual task-related properties of body state to the role of "noise" felt as unmodeled "load" perturbations on independent motor shafts. We turn to the mechanical design of DynoClimber to demonstrate effective climbing as long as its legs are maintained in a roughly antiphase relationship. In further defense of our coarse abstraction we observe that these models are sufficiently complex that so far the only analytical results for work-directed controllers encompassing physical actuator models explicitly coupled to the physical body state model have been obtained for one degree of freedom bodies (e.g. such as [53]) and that we see the present analysis as a first step along the way to that more informative but far less tractable problem. We also observe that no smooth workdirected scheme has heretofore been shown to converge even on $\mathbb{T}^{2}$

4) Coordination controller definition: Formally, letting $V:=\left(V_{1}, V_{2}\right)$ be the voltage command signal and $\delta:=\theta_{1}-\theta_{2}$ we take

$$
V(\Theta)=V_{\text {Max }}\left[\begin{array}{l}
1 \\
1
\end{array}\right]-h(\delta) \cdot\left[\begin{array}{c}
\mathrm{u} \circ \sin (-\delta) \\
\mathrm{u} \circ \sin (\delta)
\end{array}\right]
$$

where the unit step function, $\mathrm{u}$, outputs the scalar value 1 if its argument is positive and outputs 0 elsewhere, while $h: \mathbb{S}^{1} \rightarrow$ $\mathbb{R}^{1}$ is any smooth, even, positive function that vanishes if and only if its argument is 0 or $\pi$.

Combining controller and plant, our system is

$$
k_{2} \ddot{\Theta}+k_{1} \dot{\Theta}=V_{\text {Max }}\left[\begin{array}{l}
1 \\
1
\end{array}\right]-h(\delta) \cdot\left[\begin{array}{c}
\mathrm{u} \circ \sin (-\delta) \\
\mathrm{u} \circ \sin (\delta)
\end{array}\right]
$$

To verify that our control input is smooth, we show that our term containing step functions,

$$
v(\delta)=h(\delta) \cdot\left[\begin{array}{c}
\mathrm{u} \circ \sin (-\delta) \\
\mathrm{u} \circ \sin (\delta)
\end{array}\right]
$$

is differentiable. First, for $\delta \in(0, \pi))$, noting that $\mathrm{u} \circ \sin (\delta)=$ 1 and $\mathrm{u} \circ \sin (-\delta)=0$,

$$
\left.\frac{d v}{d \delta}\right|_{\delta \in(0, \pi)}=d h / d \delta \cdot\left[\begin{array}{l}
0 \\
1
\end{array}\right]
$$

and for $\delta \in(-\pi, 0)$, similarly,

$$
\left.\frac{d v}{d \delta}\right|_{\delta \in(-\pi, 0)}=d h / d \delta \cdot\left[\begin{array}{l}
1 \\
0
\end{array}\right]
$$

Because $h$ is nonnegative and smooth with isolated zeroes when its argument is 0 or $\pi, v(0)=v(\pi)=0$, and $d h / d \delta \rightarrow$ 0 as $\delta \rightarrow 0$ or $\pi$ from either side. Since the derivative of a step function is undefined at 0 , we define $(d v / d \delta)(0)=$ $(d v / d \delta)(\pi)=0$. This makes $d v / d \delta$ continuous everywhere and demonstrates that our control input is smooth despite the presence of step functions.

A final informal observation about our controller: since the controller specifies motor voltages directly, it keeps at least one motor operating along its speed-torque curve at all times. Our present implementation provides no guarantee that motor current will not fall below the motors' sustainable current ratings; using this control framework to specify voltages, as we have done here, can indeed require the motors to overheat. DynoClimber has not exhibited thermal problems, a trait we discuss in Section VI-A.

5) Proof of Correctness: For $\alpha \in \mathbb{S}^{1}$ denote the $\alpha$-translate of the diagonal in $\mathbb{T}^{2}$ as

$$
\Delta_{\alpha}:=\left\{(\theta, \theta+\alpha) \mid \theta \in \mathbb{S}^{1}\right\} .
$$

Proposition 4: The anti-diagonal tangent space,

$$
T \Delta_{\pi}:=\left\{\left(\theta_{1}, \theta_{2}, \dot{\theta}_{1}, \dot{\theta}_{2}\right) \mid \theta_{1}=\theta_{2}+\pi, \dot{\theta}_{1}=\dot{\theta}_{2}\right\}
$$

is an attracting invariant set whose domain includes $T\left(\mathbb{T}^{2}\right)$ $T \Delta_{0}$. 
Proof: Rewrite (22) in the new coordinates,

$$
\left[\begin{array}{l}
\rho_{1} \\
\rho_{2}
\end{array}\right]=\left[\begin{array}{c}
\theta_{1}-\theta_{2} \\
\theta_{1}+\theta_{2}
\end{array}\right]
$$

yielding

$$
\left[\begin{array}{l}
V_{1}(\Theta) \\
V_{2}(\Theta)
\end{array}\right]=\left[\begin{array}{c}
\ddot{\rho}_{1}+\ddot{\rho}_{2} \\
\frac{\ddot{\rho}_{2}-\ddot{\rho}_{1}}{2}
\end{array}\right] k_{2}+\left[\begin{array}{c}
\frac{\dot{\rho}_{1}+\dot{\rho}_{2}}{2} \\
\frac{\dot{\rho}_{2}-\dot{\rho}_{1}}{2}
\end{array}\right] k_{1}
$$

Solving for $\ddot{\rho}_{2}$ in the second equation, substituting it into the first, and simplifying yields

$$
\begin{aligned}
\ddot{\rho}_{1} k_{2}+\dot{\rho}_{1} k_{1}= & -h\left(\rho_{1}\right) \cdot \mathrm{u} \circ \sin \left(-\rho_{1}\right) \\
& +h\left(\rho_{1}\right) \cdot \mathrm{u} \circ \sin \left(\rho_{1}\right) \\
\ddot{\rho}_{2} k_{2}+\dot{\rho}_{2} k_{1}= & V_{1}(\Theta)+V_{2}(\Theta)
\end{aligned}
$$

Noting that $\rho_{1}$ is decoupled from $\rho_{2}$, we introduce a LaSalle function over $T \mathbb{S}^{1}$,

$$
\begin{gathered}
E\left(\rho_{1}, \dot{\rho}_{1}\right)=k_{2} \cdot \frac{\dot{\rho}_{1}^{2}}{2}-H\left(\rho_{1}\right) ; \\
H\left(\rho_{1}\right):=\int_{0}^{\left|\rho_{1}\right|} h(x) d x
\end{gathered}
$$

$h\left(\rho_{1}\right)$ goes to 0 smoothly as $\rho_{1} \rightarrow 0$, so $H$ is smooth. By construction, $h(x)>0 \forall x \in \mathbb{S}^{1}-\{0, \pi\}$, and $h(0)=h(\pi)=$ 0. $H\left(\rho_{1}\right)$ is strictly decreasing in $\left|\rho_{1}\right|$, and therefore takes its minimum at $\pi$ and its maximum at 0 , with no other critical point. It follows that $(\pi, 0)$ is the unique minimum of $H$.

Taking the time derivative of $E$ along the motions of the system, and recalling that $h(\cdot)$ is an even function, we find

$$
\begin{gathered}
\dot{E}\left(\rho_{1}, \dot{\rho}_{1}\right)= \\
k_{2} \dot{\rho}_{1} \ddot{\rho}_{1}+h\left(\rho_{1}\right) \dot{\rho}_{1}\left(\mathrm{u} \circ \sin \left(-\rho_{1}\right)-\mathrm{u} \circ \sin \left(\rho_{1}\right)\right)
\end{gathered}
$$

After substituting $\ddot{\rho}_{1}$ from (25) and cancelling terms, we obtain

$$
\dot{E}\left(\rho_{1}, \dot{\rho}_{1}\right)=-k_{1} \dot{\rho}_{1}^{2}
$$

Thus, $\dot{E}$ is negative semidefinite and $E$ is a suitable LaSalle function.

Examining the inverse image,

$$
\dot{E}^{-1}(0)=\left\{\left(\rho_{1}, 0\right) \mid \rho_{1} \in \mathbb{S}^{1}\right\}
$$

we find the only invariant subsets of $\dot{E}^{-1}(0)$ occur at the zero section corresponding to the critical points of $H$, i.e., when $\dot{\rho}_{1}=0$ and $h\left(\rho_{1}\right)=0$, which implies that $\rho_{1}=0$ or $\rho_{1}=\pi$. Since $(\pi, 0)$ is a minimum of $E$, while $(0,0)$ maximizes $E$ in $\rho_{1}$, the former is an attractor and the latter a repellor, and the result follows.

Corollary 2: The restriction dynamics on the attracting invariant submanifold $T \Delta_{\pi} \approx T \mathbb{S}^{1}$ gives rise to an almost globally asymptotically stable limit cycle.

Proof:

On $T \Delta_{\pi}$ we have $\left(\rho_{1}, \dot{\rho}_{1}\right)=(\pi, 0)$, hence, the restriction dynamics are given by $\ddot{\rho}_{2} k_{2}+\dot{\rho}_{2} k_{1}=2 V_{M a x}$, and the system yields a single attracting limit cycle of the form

$$
\left(\rho_{2}, \dot{\rho}_{2}\right)(t)=\left(\rho_{2}(0)+\omega t, \omega\right)
$$

where $\omega:=2 V_{\operatorname{Max}} / k_{1}$.

\section{Robot Design and Physical Implementation}

The initial physical adaptation of this model was intended to investigate the efficacy of the proposed template when anchored in a physical machine subject to realistically available power density at a utilitarian scale. To maintain a focus on the template dynamics, we sought to decouple the vertical and lateral climbing motions from other key environmental interactions required for climbing, such as adhesion to the wall and roll dynamics. While we initially considered using electromagnets or a substrate engineered to guarantee attachment, we feared that it would hinder our eventual goal of integrating dynamics into a versatile climbing robot. We chose instead to work with claw-like feet on a carpet substrate, a combination that proved effective as a starting point for RiSE v1.0 [9], and on which that robot's fastest climbs have been recorded [61]. This initial setting gives us confidence that the attachment developments that have enabled RiSE to move from carpet to brick, stucco, concrete, etc. [51, 18] may be adaptable to our dynamic climber as well. It also provides for an equitable comparison of the robots' relative performances.

\section{A. Mechanical Structure and Design}

The basic mechanical design is adapted directly from the two-dimensional simulation described in Section IV, which is comprised of a rigid body and two linearly-moving hands with springs. The resulting robot, depicted in Fig. 12, features two motors, each driving a crank-slider mechanism attached to an arm. As in simulation, each leg has an energy-storage spring in parallel with the crank-slider. Each foot also features a pair of passive-wrist springs which act in series with the drive actuation. These passively connect the claw to the arm and are extended during the beginning of the stance phase, acting to mitigate the loading forces on the robot. ${ }^{10}$ Heavy components such as the motors and electronics are located below the cranks in order to position the center of mass lower in allegiance to the idealized mass distribution of the template. The frame of the robot was initially constructed from ABS plastic, and later from machined aluminum. The transmission system is comprised of a bevel gear pair, a pulley pair, sliders (steel shafts and linear bearings), and aluminum links. The sprawl angles of both arms are adjustable with several presettings, including the setting of $10^{\circ}$, which corresponds to the effective sprawl angle used by geckos and cockroaches. The robot's physical parameters are summarized in Table III.

To minimize rolling, we outfitted the robot with a rollstabilization bar, as seen in Fig. 12. This bar extends laterally $20 \mathrm{~cm}$ on both sides and approximates the function of multiple legs in reducing roll dynamics. The template only considers motions in the climber's frontal plane; the roll-stabilization bar is implemented in an attempt to enforce that assumption. In future work, this bar could be replaced by active rear legs designed to apply forces normal to the wall which counteract the robot's natural rolling dynamics.

\footnotetext{
${ }^{10}$ We do not implement a damper in parallel with the wrist spring as employed in both the template and design simulation. We suspect that this damper may be important for the stability of a numerical solver, but not critical to the stability of an instantiated machine. No data or anecdotal evidence from the robot climbing indicates that the lack of a wrist damper is problematic.
} 


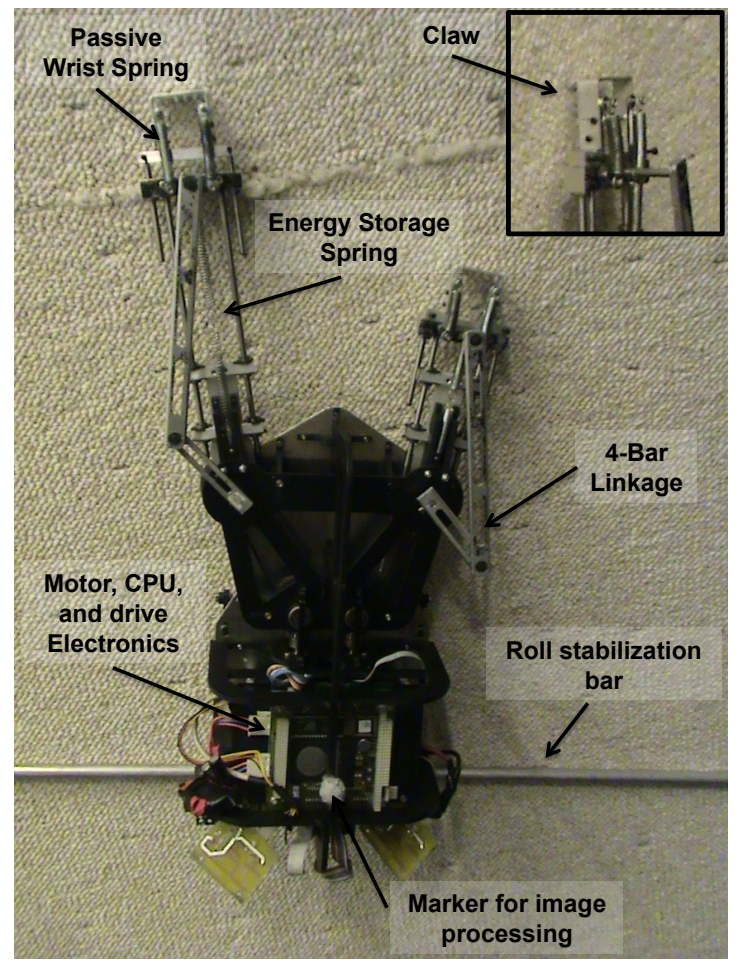

Fig. 12. An annotated picture of the modified DynoClimber hanging on the climbing track.

TABLE III

Physical Parameters Common to Both Initial and Modified ROBOT VERSIONS

\begin{tabular}{|l|l|}
\hline Body size & $400 \times 116 \times 70 \mathrm{~mm}$ (excluding cables) \\
Wrist spring stiffness & $640 \mathrm{~N} / \mathrm{m}$ \\
Parallel spring stiffness & $58 \mathrm{~N} / \mathrm{m}$ \\
Motor & Maxon RE 25 118752 \\
Gear head & Maxon Planetary Gearhead GP 32A 114473 \\
& $33: 1$ Gear ratio \\
Encoder & Maxon digital encoder HEDS55 110515 \\
& 500 count/turn \\
Leg sprawl angle & $10^{\circ}$ from centerline \\
\hline
\end{tabular}

We chose to implement a passive attachment strategy where the claw is rigidly attached to the hand. The bent teeth of the claw, shown in Fig. 12, provide a simple mechanical implementation of directional attachment, in that they engage the substrate when the leg is being pulled down, and releases when pushed up. A slight pitch angle introduced by a block under the tail of the robot ensures that the extended foot is closer to the wall than the retracted foot and aids in attachment at the expense of a slightly reduced effective stride length. While simple and generally effective, the fully passive nature of this attachment mechanism does, on occasion, result in lost footholds. ${ }^{11}$ Future foot designs include an actuator at the hand which will improve the reliability of attachment, and provide for control of the phasing of attachment and detachment.

\footnotetext{
${ }^{11}$ Most attachment failures are caused by inadequate roll stabilization: the robot occasionally rolls in such a way as to lift its flight leg slightly from the wall. If that lift coincides with the beginning of a leg's stance phase, the passive attachment mechanism permits no recourse and the foot is unable to find purchase.
}

The resulting robot was, due to alterations to the electronics which increased their mass and changes in the design to increase the structure's rigidity and strength, $20 \%$ heavier than projected. This initial climber, despite achieving record climbing speed at the time, had a number of mechanical and electrical flaws. Its ABS plastic baseplate flexed enough to allow occasional drive belt slippage, and the motor driver filtering circuitry did not have a sufficiently low break frequency, allowing too much ripple with a supply of more than $18 \mathrm{~V}$. Due to concerns about the weight of the robot, structural strength of the body, and the limits of the early version of the power electronics, the robot was initially designed to climb at a reduced speed. This speed reduction was implemented via a gear reduction of the motor of 66:1 rather than the 50:1 ratio originally determined to be optimal through simulation. After testing, however, it became clear that the robot's locomotion would be substantially more dynamic if its gear ratio were reduced.

In order to overcome the shortcomings of the initial version of the hardware a modified version of the robot featuring a more robust mechanical structure and an updated electronics infrastructure capable of operating in the $24 \mathrm{~V}+$ voltage range was built. The alterations included an all new aluminum frame and new motor drive electronics. These modifications, however, added an additional $200 \mathrm{~g}$ to the robot, resulting in a new total mass of $2.6 \mathrm{~kg}$. In light of these changes to the robot it was calculated that a new gear reduction would be necessary to optimize the robot's climbing speed. Simulation studies indicated that a total gear reduction of 56:1, which is closer to the original design specification, would maximize climbing speed. Prospective changes were prototyped in the design model by a literal transcription of parameters, though due to the speed limitations of the simulation engine, the design model could not be tested for a comprehensive set of parameter variations. Instead, the vertical power stroke model was simulated over large gear ratio (altering $F_{s}$ and $l_{s}$ reciprocally in the model) and stride-length ranges (direct changes to $l_{s}$ ) to determine roughly-optimal gear ratio choices. Two criteria were used to determine optimality: the design choices needed to optimize climbing speed while ensuring that steady-state climbing occurred with the motors drawing less than their continuous current ratings. ${ }^{12}$

Comparison of power stroke model and robot: In Table IV, we give the parameters used in both versions of the physical robot (see Section VI-A for a more complete description) and the best physical equivalences for the vertical power stroke model. Table II verifies that both parameter sets meet the criteria of our proof of stability.

Moreover, we can see that the continuously permissible force generated in both versions of the vertical power stroke model is, indeed larger than the weight of the climber. Thus, the constant voltage controller and the current-limiting controller from Section IV-C.1 would both cause the vertical power stroke to converge to the same steady-state behavior.

\footnotetext{
${ }^{12}$ Exact design choices are often determined by readily-available part sizes; the gear ratio modification from the initial to the modified version of DynoClimbers was physically implemented by changing the belt gear ratio from 1:1 to $12: 14$, for instance.
} 
TABLE IV

VERTICAL POWER STROKE MODEL AND ROBOT PARAMETERS

\begin{tabular}{|c|c|c|c|c|c|c|}
\hline Parameter & Description & Units & Initial Robot & Modified Robot & Initial Power Stroke & Modified Power Stroke \\
\hline$M$ & Body mass & $k g$ & 2.4 & 2.6 & 2.4 & 2.6 \\
\hline$l_{s}$ & Stroke length & $m$ & 0.12 & 0.13 & 0.12 & 0.13 \\
\hline$V$ & Battery Voltage & $V$ & 18 & 30 & 18 & 30 \\
\hline$r$ & Pinion gear radius & $m$ & $\cdot$ & . & .0382 & .0414 \\
\hline$l_{c}$ & Crank length & $m$ & 6 & .65 & $\cdot$ & $\cdot$ \\
\hline$G$ & Gear ratio & $\cdot$ & $66: 1$ & $56.6: 1$ & $66: 1$ & $56.6: 1$ \\
\hline$k$ & Energy storage spring & $N / m$ & 58 & 58 & 58 & 58 \\
\hline$F_{s}$ & Output stall force & $N$ & . & . & 403 & 531 \\
\hline$\tau_{s}$ & Motor stall torque & $N m$ & .233 & .388 & .233 & .388 \\
\hline$v_{n l}$ & Output no-load speed & $\mathrm{m} / \mathrm{s}$ & . & $\cdot$ & .62 & 1.30 \\
\hline$\omega_{n l}$ & Motor no-load speed & $\mathrm{rad} / \mathrm{s}$ & 1068 & 1780 & 1068 & 1780 \\
\hline$F_{c}$ & Output continuous force & $N$ & $\cdot$ & $\cdot$ & 41.5 & 32.81 \\
\hline$\tau_{c}$ & Motor continuous torque & $N m$ & .0240 & .0240 & .0240 & .0240 \\
\hline & Controller & $\cdot$ & Mirror-law (16) & SEWD (21) & Constant voltage & Constant voltage \\
\hline
\end{tabular}

\section{B. Experimental Setup and Procedure}

To evaluate the robot climbing performance, a $4 \mathrm{~m} \times 0.8 \mathrm{~m}$ carpet-surface vertical climbing wall was built, part of which is shown in Fig. 12. A commercial 6-axis force sensor (AMTI HE6x6) was built into a panel of the wall to collect interacting forces between the left foot of the robot and the wall. A vision system composed of a commercial HD video camera (SONY HDR-SR1) and two spotlights for robot motion tracking is located $4 \mathrm{~m}$ away facing the climbing wall. In order to simplify the off-line analysis of the visual data, the robot is painted black and 2 markers are installed rigidly on the body for size calibration.

Both force data and video data are collected while the robot climbs. Video streams are exported into sequential images for post processing in Matlab. Each color image is converted to black and white by setting threshold empirically and the "white" objects in the image are distinguished from each other by a labeling function and by their geometric relations. For each run the robot was started from rest at the bottom of the track and the last 5 seconds of climbing were analyzed. In this section we describe experimental results from two versions of the robot.

\section{Initial Robot Performance}

Figure 13 shows the trajectory and velocity of the center of mass of the robot while climbing. This figure shows the final 16 steps (8 per leg) after the robot had accelerated from rest to a steady-state climbing speed. Subplots (a) and (c) show the vertical velocity oscillates substantially during each stride, but averages to about $30 \mathrm{~cm} / \mathrm{s}$ as the robot climbs with a stride frequency of $1.8 \mathrm{~Hz}$. Figure 14 compares the ground reaction forces and velocities of the robot and a simulation of a similar $2.4 \mathrm{~kg}$ version which has been modified to match the larger gear reduction and lower applied voltage used in the experiments shown in Fig. 13.

The lateral position and velocity, shown in Fig. 13, subplots (b) and (d) indicate that the lateral oscillations are larger than the animal-inspired template, with lateral velocities about 1.5 times as large as the scaled template. As predicted by simulation, the lower stride frequency results in larger lateral swings with each stride. The pendular frequency of the original
A

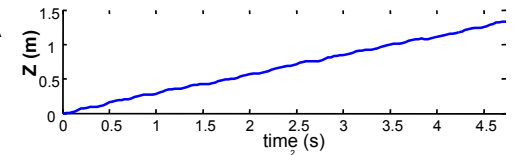

B

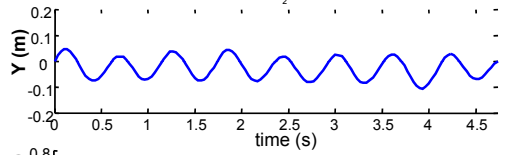

$\mathrm{C} \overrightarrow{\mathrm{\omega}}^{0}$
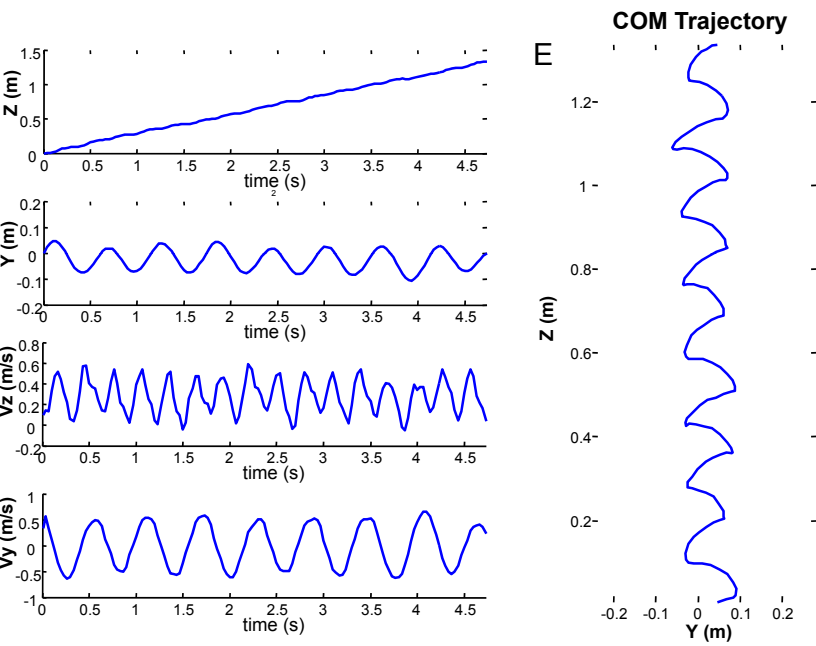

Fig. 13. Video-based marker data for eight strides of the robot climbing. (a) vertical displacement, (b) lateral displacement, (c) vertical velocity, (d) lateral velocity, and (e) the path of the center of mass during climbing.

template body is generally slower than the template's stride frequency. However, as stride frequencies are reduced and therefore near the pendular frequency, the rotational motion of the climber becomes dramatically more pronounced. The COM position plot (e), shows how at the end of each stride the motion of the robot is largely lateral. With each new foot placement the robot begins to reverse direction and accelerate upwards again.

The top row of Fig. 14 shows the magnitude and direction of the ground reaction forces for both the $2.4 \mathrm{~kg}$ simulation and the left foot of the $2.4 \mathrm{~kg}$ robot. Due to the location of the force plate on the climbing wall, only force data for the left foot is available. The robot's legs remain attached for slightly longer each stride than predicted by simulation due to the existence of a double support phase: using the passive attachment mechanism, a foot is disengaged from the substrate after the other foot has engaged and begun pulling up the robot. Consequently, slightly larger magnitude attachment forces and foot-attachment duty factors of about $60 \%$ are seen on the robot.

The second and third rows show the traces of the vertical 
and lateral speed, which are very close in both magnitude and phasing. The experimental velocity data's coarseness stems from the limited frame rate of the camera used to record the motion. While the simulation climbs slightly faster than the robot it does not incorporate artifacts associated with attaching and detaching from a compliant substrate or friction of the body against that substrate.
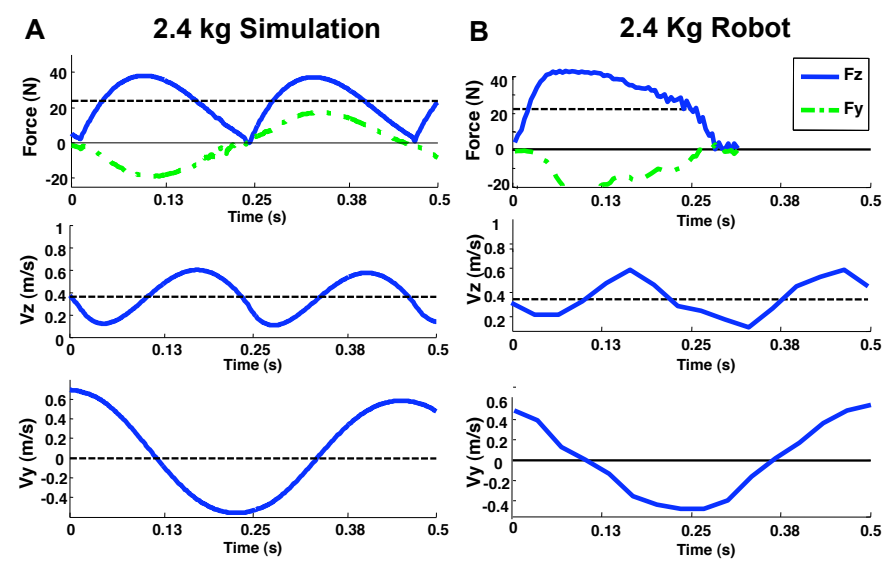

Fig. 14. velocities for the $2.4 \mathrm{~kg}$ simulation and robot. Note: due to the location of the force plate, only forces for the left foot of the robot were measured.

These initial results demonstrate that fast, dynamically stable vertical running with a template-inspired bipedal climbing robot is achievable. Furthermore, an examination of the stride dynamics indicates that the robot successfully recreates the lateral motions and ground reaction force patterns seen in the template and in the animals. The self-stabilizing nature of the resulting gait is highlighted in the first video attachment where the robot is shown quickly recovering from a missed foothold near the top of its climb.

Past tradition in the locomotion literature has been to provide qualitative comparison of animal, simulation, and robot test trajectories as we do here in assessing the quality of model fit $[29,42]$. Newer statistical methods of phase comparison [62] suggest a much more intensive data set than presently available in our prototype implementation can deliver a far more precise estimate of fit, but such a statistical comparison lies beyond the scope of the present paper. The comparison of the robot's motion with the simulation also demonstrates good correspondence, with the average upward velocity, peak lateral velocities, and peak ground reaction forces agreeing to within about $10 \%$. In addition, the phasing between lateral and vertical motions and the ratios of the lateral and vertical forces are all preserved.

\section{Modified Robot Performance}

As described in Section VI-A, the electrical and mechanical limitations in the original design induced the design and construction of a modified version of the robot weighing $2.6 \mathrm{~kg}$. The results of running the robot with these design changes and the self-excited, work-directed control strategy (see Section V-B.2) are shown in Fig. 15. With a 20V supply
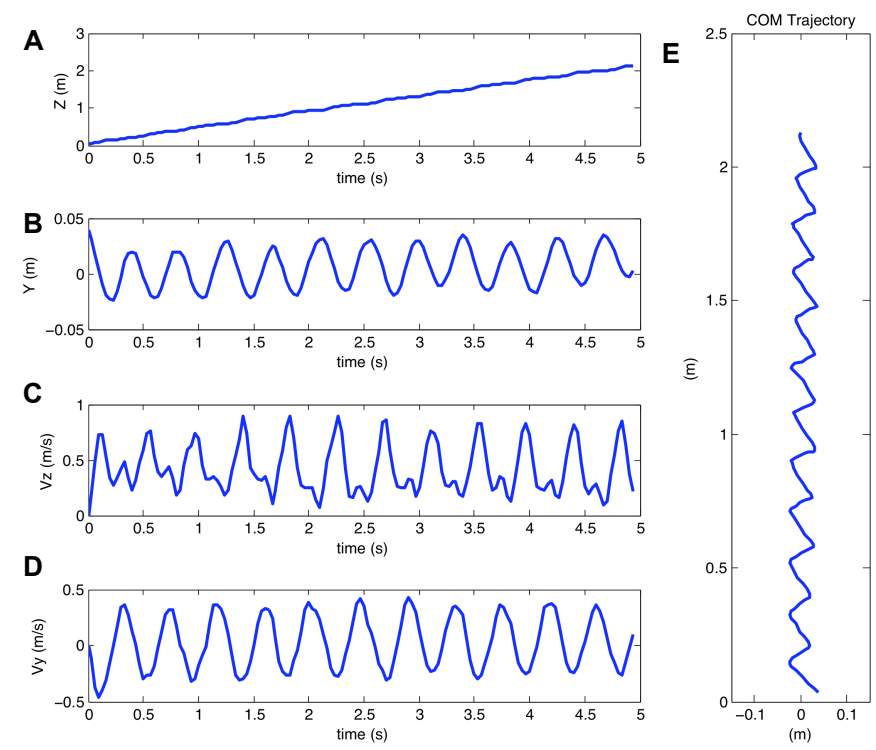

Fig. 15. Video-based marker data for eleven strides of the modified robot climbing with a $20 \mathrm{~V}$ supply. (a) vertical displacement, (b) lateral displacement, (c) vertical velocity, (d) lateral velocity, and (e) the path of the center of mass during climbing.

the robot ran at an average speed of $39 \mathrm{~cm} / \mathrm{s}$. As shown in Fig. 15(d), the amplitude of the lateral velocity oscillations was reduced from $0.6 \mathrm{~m} / \mathrm{s}$ to $0.4 \mathrm{~m} / \mathrm{s}$, matching the scaled animal-inspired template, see Fig. 3. However, as is evident from Fig. 15(c) and (e), the vertical climbing motion is now asymmetric. The robot limps as it climbs, pulling mostly to the right with one step and then upwards and to the left with the next step. A more detailed view of a single limping stride is shown in Fig. 16.

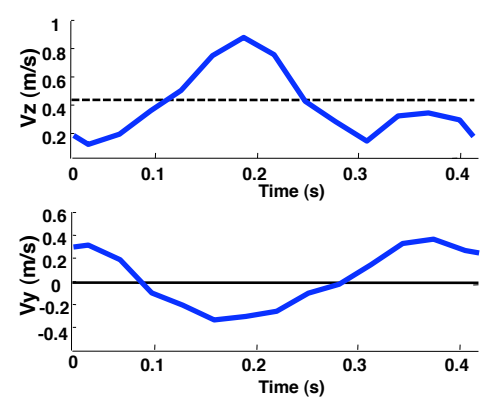

Fig. 16. Vertical (Vz) and horizontal (Vy) COM velocities for the $2.6 \mathrm{~kg}$ robot with a limping gait.

This version of the physical platform demonstrates a repetitive, asymmetric period-2 gait with no obvious relationship to initial conditions, while the design simulation has not demonstrated any such asymmetrical behavior. Identifying more precisely the root of these phenomena using simplified climbing models is a topic of ongoing research, but outside the scope of this paper.

In order to test the performance capabilities of the robot, it was run with an input voltage set to $20 \mathrm{~V}, 25 \mathrm{~V}$, and $32 \mathrm{~V}$. We found that that average speed of climbing for a $20 \mathrm{~V}$ input was $38.8+/-2.8 \mathrm{~cm} / \mathrm{s}$, and when we increase the input 
voltage to $25 \mathrm{~V}$ the robot climbs at $44.7+/-1.5 \mathrm{~cm} / \mathrm{s}$. With a $32 \mathrm{~V}$ supply the robot did not demonstrate a period-2 gait and ran at a record breaking $66 \mathrm{~cm} / \mathrm{s}$, which in both an absolute and in a dynamically scale-independent sense is faster than the cockroach climbing that inspired the dynamic climbing template. Climbing at this speed is shown in the second video attachment.

In the short term, electronic infrastructure provides the practical limit on the maximum voltage that can be applied. Note that electromagnetic motors are only thermally affected by motor current; voltages larger than nominal are still "safe" as long as motor current is kept to permissible values and the motor does not spin faster than its specified peak operational speed. As voltage is increased, additional care must be taken to ensure these criteria are met. The robot's legs must be loaded carefully, with neither too much nor too little resistance. Running the robot's legs at a high voltage with a heavy load is likely to cause the motors to draw excessive current and overheat, while spinning the motors unloaded could permit them to exceed the motor's maximum permissible speed. However, for a range of acceptable motor speeds, this increase in voltage enables an impressive increase in power output.

The ability to sustain climbing at these velocities and voltage levels for extended periods is uncertain, but the need for high-speed vertical climbing is typically intermittent. For example, even if due to motor thermal constraints this speed could only be maintained for one minute, the robot would still be able to scale a height of 40 meters before stopping.

\section{E. Discussion of Results}

With our spring-assisted dynamic climbing robot, DynoClimber, we have developed and implemented an operational platform design that exploits bioinspired center of mass motions and ground reaction forces to produce vertical climbing speeds of over one and a half body-lengths per second. For symmetric gaits, the robot performance in terms of climbing speed, ground reaction force profiles, and velocity phasing match the predictions of the dynamic simulation. See Table V for a comparison of robot and model, as well as scaled animal, climbing speeds. This agreement suggests that the argument in Section IV-D is correct in predicting that we are near the largest and fastest climber that can implement template-based climbing dynamics with the currently employed off-the-shelf motors.

In both cases, the physical platform is roughly $30 \%$ slower than the corresponding models. This result is unsurprising given the frictionless environment and perfect attachment of the simulation models. However, the models' abilities to anticipate rough performance trends is well brought out in the table: both come close to predicting the $223 \%$ empirical increase in steady state climbing speed (the design model predicts a $220 \%$ increase while the vertical power stroke model predicts a $227 \%$ increase).

In this regard, we find the rough agreement between the design and vertical power stroke models particularly notable: despite the design model's relative complexity (rotational dynamics, crank-slider transmission, wrist springs, and rigid body dynamics), the greatly simplified purely-vertical power stroke model climbs within $10 \%$ of the design model's speed. The small discrepancy between these vastly differently abstracted models suggests the extent to which a climber's power train determines its vertical velocity.

This agreement reinforces our use of simplified models to make first-order design decisions. While a design model is required to support crucial decisions bearing on implementation details (changing the robot's mass-distribution, for instance), overarching questions concerning the soundness of the power stroke concept and its impact on vertical climbing behavior can be explored far more thoroughly (and, indeed, with some consequent mathematical guarantees) using the vertical power stroke model. Thus, the introduction of this coarse, reducedorder model allows us simulate power train design choices (i.e. motor choice, gear ratio, or stride length) much more extensively than would be possible with the high-fidelity design model alone. Moreover, provided that the unmodeled dynamics are stable and not disruptive, that model delivers equivalent results.

TABLE V

CLIMBER AND MODEL PERFORMANCE

\begin{tabular}{|l|c|c|}
\hline Parameter & Initial Climber & Mod. Climber \\
\hline Sim. Speed: Fig. 8 (Sec. IV-C.3) & $0.44 m / s$ & $0.85 \mathrm{~m} / \mathrm{s}$ \\
Power Stroke Speed: $\bar{\zeta}^{*}(15)$ & $0.41 \mathrm{~m} / \mathrm{s}$ & $0.93 \mathrm{~m} / \mathrm{s}$ \\
Robot Climbing Speed & $0.30 \mathrm{~m} / \mathrm{s}$ & $0.67 \mathrm{~m} / \mathrm{s}$ \\
\hline Scaled Roach [19] & $.61 \mathrm{~m} / \mathrm{s}$ & $.62 \mathrm{~m} / \mathrm{s}$ \\
\hline
\end{tabular}

The robot's climbing speed is hindered substantially if it exhibits a limping gait, and both simulation models lose some, but not all, predictive ability. For instance, at $20 \mathrm{~V}$, the vertical power stroke model predicts a climbing speed of $56 \mathrm{~cm} / \mathrm{s}, 44 \%$ above the robot's $39 \mathrm{~cm} / \mathrm{s}$ vertical speed; at $25 \mathrm{~V}$ it suggests the climber will travel at $71 \mathrm{~cm} / \mathrm{s}, 58 \%$ above the climber's vertical speed. ${ }^{13}$ The error in correspondence is due to the pernicious effects of the period-2 gait demonstrated by DynoClimber; we would expect substantially improved predictive value (in line with the results from Table V) were the climber to have climbed with a period-1 gait at lower voltages. As mentioned earlier, our morphologically and parametrically accurate design model does not demonstrate a period-2 or higher gait; assessing the cause of DynoClimber's limp is beyond the scope of this paper and the subject of ongoing research.

To determine the "scaled roach" vertical speed values, we applied the scaling laws derived in Section III to the measured cockroach mass and speed from [19] Moreover, while the robot and both simulations indicate that our initial climber was incapable of achieving scaled-template-like vertical speeds, the modified climber is able to do so in both simulation and the physical world.

\section{CONCLUSION AND FUtURE WORK}

Biologists' discovery of a template [20] — a common dynamical pattern — for climbing in diverse animal species

\footnotetext{
${ }^{13}$ While a limp was demonstrated at $20 \mathrm{~V}$ and $25 \mathrm{~V}$, but not $32 \mathrm{~V}$, we believe that the robot's propensity to limp was not a strict function of applied voltage. Indeed, limps appeared during informal testing at each of the voltage levels.
} 
[19] inspired our adaptation of their simulation model, resulting in a physically instantiated, bipedal, dynamic vertical climber. Systematic scaling arguments along with numerical and mathematical modeling result in a robot that exhibits a strong correspondence to the original template model despite accommodations to the exigencies of commercially available prime movers. The addition of a force-assist spring in parallel with the actuator in the legs and the switch to a force controlled actuation scheme allow conventionally actuated legged robotic climbing at animal-like speeds, even approaching the upper limits of commercially available power densities. In addition, it appears that the characteristic force and motion patterns of the animals and the steady gaits exhibited by the template are reproducible in the physically anchored version, the robot.

In addition to this constructive demonstration that the FullGoldman template [19] dynamics can be successfully anchored in a synthetic dynamic climber, this paper has presented an analysis of the internal vertical dynamics of the powerstroke system and shown that it is inherently stable. This, coupled with the development and proof of stability of the gait coordination controller, has laid an analytical foundation for the study of vertical dynamic climbing.

Future theoretical work, however, will be required to fully understand how the robot's physical structure and control scheme interact to determine its lateral stability. This design study motivates more practical empirical work investigating the effect of increased climbing speed on the attachment and detachment of feet capable of adhering to smooth surfaces. In addition to enabling higher speeds, proper exploitation of the locomotion dynamics could also lead to greater maneuverability without having to increase the kinematic complexity of the design. Eventually, however, the body dynamics that result in fast, stable climbing will be integrated into robots with more limbs to achieve a truly utilitarian dynamical robot that can operate on a number of surfaces and on substrates with varied geometries.

Accumulating simulation results [19, 63] support the hypothesis that lateral and rotational stability for climbers is improved by generation of large lateral inpulling forces. Testing this hypothesis in a physical platform entails studying DynoClimber's response to controlled perturbations for different sprawl angles. Our hypothesis suggests that for some sprawl angle larger than 0, the robot's settling time following perturbations should be minimized.

In addition to future experimental work to examine the effect of leg sprawl angle on vertical climbing, this platform could be used to investigate why animals alter their horizontal force generation patterns as the inclination of the substrate changes from horizontal to vertical.

In conclusion, we have built the first dynamical vertical climbing robot. The successful scaling and implementation of the bio-inspired template has enabled us to explore the possible advantages of this novel design that uses passivedynamic elements. Because the robot exhibits a scaled version of the dynamics measured in diverse animal species, we are also hopeful that it might be useful as a physical model to generate a next round of testable hypotheses that might lead to new discoveries in animal climbing.

\section{APPENDIX - DERIVATION OF THE RETURn MAP}

Writing down the closed form of the return map requires us to evaluate the flow of the system at an implicitly defined time. Since the system is affine, its flow can be readily determined from its differential form, first stated in eq. 5:

$$
\dot{z}=A z+b .
$$

Additionally, the initial conditions of the system are defined to be $z_{0}=:(0, \zeta)$. Using these initial conditions, the system's flow can be readily written as:

$$
f^{t}(z)=e^{A t} z_{0}-A^{-1} b
$$

The time to flow from $\mathcal{L}_{1}$ by means of $f^{t}$ to $\tilde{\mathcal{L}}_{1}$ is once again a function of the initial condition on the section, $\zeta \in \mathcal{L}_{1}$ :

$$
T(\zeta)=\min \left\{t>0 \mid \ell_{1} \circ f^{t} \ell_{2}^{\dagger}(\zeta)=l_{s}\right\},
$$

Constrained operation along the speed-torque curve of the motor force $F_{m}$ in eq. 4 yields a several-term transcendental appearance of $T$, resulting in a solution for $T$ which cannot be expressed in closed form and must instead be expressed implicitly by the equation $\ell_{1} \circ f^{T} \ell_{2}^{\dagger}(\zeta)=l_{s}$.

The return map $R(\zeta)$ is then given by:

$$
\begin{array}{r}
R(\zeta)=\ell_{2} \circ s \circ f^{T} \circ \ell_{2}^{\dagger}(\zeta)= \\
\frac{-1}{2 \alpha \sqrt{\sigma^{2}-1}} \\
\left(e^{\alpha \lambda_{2} T}\left[\beta \lambda_{1}+\alpha \zeta\right] \cdot \lambda_{2}-e^{\alpha \lambda_{1} T}\left[\beta \lambda_{2}+\alpha \zeta\right] \cdot \lambda_{1}\right),
\end{array}
$$

with

$$
\lambda_{1}=-\sigma+\sqrt{\sigma^{2}-1}, \quad \lambda_{2}=-\sigma-\sqrt{\sigma^{2}-1}
$$

defined to be the eigenvalues divided by $\alpha$.

Differentiating $R$ with respect to $\zeta$ and determining $\frac{d T}{d \zeta}$ using the implicit function theorem, we arrive at

$$
D R(\zeta)=\frac{\left(2 \alpha \zeta \sqrt{\sigma^{2}-1}\right) e^{\alpha T\left(-\sigma+\sqrt{\sigma^{2}-1}\right)}}{A_{0} e^{2 \alpha T \sqrt{\sigma^{2}-1}}+A_{1}}
$$

where:

$$
\begin{aligned}
& A_{0}=\left(\beta-\alpha \sigma \zeta+\alpha \zeta \sqrt{\sigma^{2}-1}\right) \\
& A_{1}=\left(\alpha \sigma \zeta-\beta+\alpha \zeta \sqrt{\sigma^{2}-1}\right)
\end{aligned}
$$

\section{ACKNOWLEDGMENTS}

This work was supported in part by DARPA/SPAWAR Contract N66001-05-C-8025 and by NSF FIBR EF-0425878. Jonathan Clark was supported by the IC Postdoctoral Fellow Program under grant number HM158204-1-2030. We thank Bob Full and Dan Goldman for numerous discussions and insight related to the scaling and adaptation of their vertical climbing template model. We thank Haldun Komsuoglu for porting his EduBot electronics and controls hardware and software infrastructure to this platform. In addition, we would like to thank Al Rizzi, Martin Buehler, and Aaron Saunders for a number of helpful discussions bearing on design and control of the physical platform. 


\section{REFERENCES}

[1] J. E. Clark and D. E. Koditschek, "A spring assisted one degree of freedom climbing model," in Springer-Verlang, Lecture Notes on Control and Information Sciences, 2006, pp. 43-64.

[2] J. E. Clark, D. I. Goldman, T. S. Chen, R. J. Full, and D. Koditschek, "Toward a dynamic vertical climbing robot," in International Conference on Climbing and Walking Robots (CLAWAR). Brussels, Belgium: Professional Engineering Publishing, 2006, vol. 9.

[3] J. E. Clark, D. G. Goldman, P. C. Lin, G. Lynch, T. S. Chen, H. Komsuoglu, R. J. Full, and D. E. Koditschek, "Design of bio-inspired dynamical vertical climbing robot," in Robots: Science and Systems, Atlanta, GA, June 27-29 2007.

[4] G. Lynch, J. Clark, and D. Koditschek, "A self-exciting controller for high-speed vertical running," in Intelligent Robots and Systems, 2009. IROS 2009. IEEE/RSJ International Conference on. IEEE, 2009, pp. 631-638.

[5] C. Balaguer, A. Gimenez, J. Pastor, V. Padron, and C. Abderrahim, "A climbing autonomous robot for inspection applications in $3 \mathrm{~d}$ complex environments." Robotica, vol. 18, pp. 287-297, 2000.

[6] G. La Rosa, M. Messina, G. Muscato, and R. Sinatra, "A low-cost lightweight climbing robot for the inspection of vertical surfaces." Mechatronics, vol. 12, no. 1, pp. 71-96, 2002.

[7] D. Bevly, S. Dubowsky, and C. Mavroidis, "A simplified cartesiancomputed torque controller for highly geared systems and its application to an experimental climbing robot." Transactions of the ASME. Journal of Dynamic Systems, Measurement and Control, vol. 122, no. 1, pp. 27-32, 2000.

[8] T. Bretl, S. Rock, and J. C. Latombe, "Motion planning for a threelimbed climbing robot in vertical natural terrain," in IEEE International Conference on Robotic and Automotion (ICRA 2003), vol. 205, Taipei, Taiwan, 2003, pp. $2946-295$.

[9] K. Autumn, M. Buehler, M. R. Cutkosky, R. Fearing, R. Full, D. Goldman, R. Groff, W. Provancher, A. Rizzi, U. Saranli, A. Saunders, and D. Koditschek, "Robotics in scansorial environments," in Unmanned Systems Technology VII, D. W. G. G. R. Gerhart, C. M. Shoemaker, Ed., vol. 5804. SPIE, 2005, pp. 291-302.

[10] "http://www.vortexhc.com/vmrp.html."

[11] J. Xiao, A. Sadegh, M. Elliot, A. Calle, A. Persad, and H. M. Chiu, "Design of mobile robots with wall climbing capability," in Proceedings of IEEE AIM, Monterey, CA, Jul. 24-28, 2005, pp. 438-443.

[12] K. Daltorio, A. Horchler, S. Gorb, R. Ritzmann, and R. Quinn, "A small wall-walking robot with compliant, adhesive feet," in Intelligent Robots and Systems, 2005.(IROS 2005). 2005 IEEE/RSJ International Conference on. IEEE, 2005, pp. 3648-3653.

[13] M. P. Murphy and M. Sitti, "Waalbot: An agile small-scale wall-climbing robot utilizing dry elastomer adhesives," IEEE/ASME Transactions on Mechatronics, vol. 12, no. 3, pp. 330-338, 2007.

[14] M. P. Murphy, C. Kute, and M. Sitti, "Waalbot ii: Adhesion recovery and improved performance of a climbing robot using fibrillar adhesives," International Journal of Robotics Research, vol. 30, no. 1, pp. 118-133, 2011.

[15] "http://berkeley.edu/news/media/releases/2002/09/rfull/robots.html."

[16] G. A. Bekey, Autonomous Robots: From Biological Inspiration to Implementation and Control. MIT Press, 2005.

[17] A. Asbeck, S. Kim, M. Cutkosky, W. Provancher, and M. Lanzetta, "Scaling hard vertical surfaces with compliant microspine arrays," The International Journal of Robotics Research, vol. 25, no. 12, p. 1165, 2006.

[18] M. Spenko, C. Haynes, A. Saunders, M. Cutkosky, A. Rizzi, R. Full, and D. E. Koditschek, "Biologically inspired climbiing with a hexapedal robot," Journal of Field Robotics, vol. 4-5, no. 25, pp. 223-242, 2008.

[19] D. I. Goldman, T. S. Chen, D. M. Dudek, and R. J. Full, "Dynamics of rapid vertical climbing in a cockroach reveals a template," Journal of Experimental Biology, vol. 209, pp. 2990-3000, 2006.

[20] R. J. Full and D. E. Koditschek, "Templates and anchors: Neuromechanical hypotheses of legged locomotion on land," Journal of Experimental Biology, vol. 202, no. 23, pp. 3325-3332, 1999.

[21] R. Blickhan and R. J. Full, "Similarity in multilegged locomotion: Bounding like a monopod," Journal of Comparative Physiology, vol. 173, no. 5, pp. 509-517, 1993.

[22] G. A. Cavagna, N. C. Heglund, and T. C. R., "Mechanical work in terrestrial locomotion: Two basic mechanisms for minimizing energy expenditure," American Journal of Physiology, vol. 233, 1977.

[23] T. A. MCMahon and G. C. Cheng, "Mechanics of running. how does stiffness couple with speed?" Journal of Biomechanics, vol. 23, no. 1, pp. 65-78, 1990.
[24] J. Schmitt and P. Holmes, "Mechanical models for insect locomotion: Dynamics and stability in the horizontal plane i. theory," Biological Cybernetics, vol. 83, no. 6, pp. 501-515, 2000.

[25] A. Degani, A. Shapiro, H. Choset, and M. T. Mason, "A dynamic single actuator vertical climbing robot," in Proceedings, IEEE/RSH International Conference on Intelligent Robots and Systems, San Diego, CA, USA, 2007.

[26] A. Degani, S. Feng, H. B. Brown, K. M. Lynch, H. Choset, and M. T. Mason, "The parkourbot - a dynamic bowleg climbing robot," in Proceedings of the 2011 IEEE International Conference on Robotics and Automations, Shanghi, China, May 9-13, 2011.

[27] G. C. Haynes, A. Khripin, G. Lynch, A. A. R. Aaron Saunders, and D. E. Koditschek, "Rapid pole climbing with a quadrupedal robot," in Proceedings - IEEE International Conference on Robotics and Automation, 2009.

[28] W. R. Provancher, S. I. Jensen-Sgal, and M. A. Fehlberg, "Rocr: An energy-efficient dynamic wall-climbing robot," IEEE/ASME Transactions on Mechatronics, vol. PP Issue: 99, 2010.

[29] K. Autumn, S. T. Hsieh, D. M. Dudek, J. Chen, C. Chitaphan, and R. J. Full, "Dynamics of geckos running vertically," Journal of Experimental Biology, vol. 209, pp. 260-270, 2006.

[30] J. Schmitt, M. Garcia, R. Razo, P. Holmes, and R. Full, "Dynamics and stability of legged locomotion in the horizontal plane: a test case using insects.” Biological Cybernetics, vol. 86, no. 5, pp. 343-53, 2002.

[31] G. La Rosa, M. Messina, G. Muscato, and R. Sinatra, "A low-cost lightweight climbing robot for the inspection of vertical surfaces," Mechatronics, vol. 12, no. 1, pp. 71-96, 2002.

[32] P. Birkmeyer, K. Peterson, and R. Fearing, "DASH: A dynamic $16 \mathrm{~g}$ hexapedal robot," in IEEE/RSJ International Conference on Intelligent Robots and Systems, 2009. IROS 2009, 2009, pp. 2683-2689.

[33] "http://www.maxonmotorusa.com."

[34] R. M. Alexander and A. S. Jayes, "A dynamic similarity hypothesis for the gaits of quadrupedal mammals," Journal of Zoology, vol. 201, pp. $135-152,1983$

[35] J. Schmitt, M. Garcia, R. Razo, P. Holmes, and R. Full, "Dynamics and stability of legged locomotion in the horizontal plane: a test case using insects." Biological Cybernetics, vol. 86, no. 5, pp. 343-53, 2002.

[36] R. M. Alexander, Elastic Mechanisms in Animal Movement. Cambridge: Cambridge University Press, 1988.

[37] H. M. Herr, G. T. Huang, and T. A. McMahon, "A model of scale effects in mammalian quadrupedal running," The Journal of Experimental Biology, vol. 205, pp. 959-967, 2002.

[38] R. M. Alexander, Principles of Animal Locomotion. Princeton University Press, 2003.

[39] R. Kornbluh, R. Pelrine, J. Eckerle, J. Joseph, S. R. I. Int, and M. Park, Electrostrictive polymer artificial muscle actuators, 1998, vol. 3.

[40] L. C. Rome, R. P. Funke, R. M. Alexander, G. Lutz, H. Aldridge, F. Scott, and M. Freadman, "Why animals have different muscle fibre types." Nature, vol. 335, no. 6193, p. 824, 1988.

[41] J. M. Hollerbach, I. W. Hunter, and J. Ballantyne, "A comparative analysis of actuator technologies for robotics," in The Robotics Reveiw 2. The MIT Press, 1992, pp. 299-342.

[42] R. Altendorfer, N. Moore, H. Komsuoglu, M. Buehler, J. Brown H. B, D. McMordie, U. Saranli, R. Full, and D. E. Koditschek, "Rhex: A biologically inspired hexapod runner," Autonomous Robots, vol. 11, no. 3, pp. 207-213, 2001

[43] J. Weingarten, R. Groff, and D. Koditschek, "A framework for the coordination of legged robot gaits," in IEEE International Conference of Robotics, Automation and Mechatronics, Singapore, 2004, pp. 679-686.

[44] E. Z. Moore, D. Campbell, F. Grimminger, and M. Buehler, "Reliable stair climbing in the simple hexapod 'rhex'," in Proceedings - IEEE International Conference on Robotics and Automation, vol. 3, 2002, pp. 2222-2227.

[45] K. C. Galloway, G. C. Haynes, B. D. Ilhan, A. M. Johnson, R. Knopf, G. Lynch, B. Plotnick, M. White, and D. E. Koditschek, "X-rhex: A highly mobile hexapedal robot for sensorimotor tasks," University of Pennsylvania, Tech. Rep., 2010.

[46] A. De, G. Lynch, A. Johnson, and D. E. Koditschek, "Motor selection using task specifications and thermal limits," submitted to TePRA 2011.

[47] S. Kim, J. E. Clark, and M. R. Cutkosky, "isprawl: Design and tuning for high-speed autonomous open-loop running," International Journal of Robotics Research, vol. 25, no. 9, pp. 903-912, 2006.

[48] M. Boggess, R. Schroer, R. Quinn, and R. Ritzmann, "Mechanized cockroach footpaths enable cockroach-like mobility," in Proceedings of IEEE ICRA, New Orleans, LA, 2004, pp. 2871-2876.

[49] H. Komsuoglu, "Toward a formal framework for open-loop stabilization of rhythmic tasks," Ph.D. dissertation, University of Michigan, 2004. 
[50] S. Kim, A. Asbeck, M. R. Cutkosky, and W. R. Provancher, "Spinybot ii: Climbing hard walls with compliant microspines," in Proceedings, IEEE - ICAR, Seattle, WA, July 17-20, 2005.

[51] M. Spenko, M. R. Cutkosky, R. Majidi, R. Fearing, R. Groff, and K. Autumn, "Foot design and integration for bioinspired climbing robots," in Unmanned Systems Technology VIII. SPIE, 2006.

[52] P. Holmes, R. J. Full, D. E. Koditschek, and J. Guckenheimer, "The dynamics of legged locomotion: Models, analyses, and challenges," SIAM Review, vol. 48, no. 2, pp. 207-304, 2006.

[53] D. Kodistchek and M. Buehler, "Analysis of a simplified hopping robot," International Journal of Robotics Research, vol. 10, no. 6, pp. 587-605, 1991.

[54] M. H. Raibert, Legged robots that balance, ser. MIT Press series in artificial intelligence. Cambridge, Mass.: MIT Press, 1986.

[55] M. Buehler, D. E. Koditschek, and P. J. Kindlmann, "Planning and control of robotic juggling and catching tasks," International Journal of Robotics Research, vol. 13, no. 2, pp. 101-118, 1994.

[56] R. Ghigliazza, R. Altendorfer, P. Holmes, and D. Koditschek, "A simply stabilized running model," SIAM Review, vol. 47, pp. 519-549, 2005.

[57] P. Gregorio, M. Ahmadi, and M. Buehler, "Design, control, and energetics of an electrically actuated legged robot," IEEE Transactions on Systems, Man, and Cybernetics, Part B, vol. 27, no. 4, pp. 626-634, 1997.

[58] J. Guckenheimer and P. Holmes, Nonlinear Oscillations, Dynamical Systems, and Bifurcations of Vector Fields. Springer, 1983.

[59] M. Buehler, D. E. Koditschek, and P. J. Kindlmann, "A simple juggling robot: Theory and experimentation," in Experimental Robotics I. Spring-Verlag, 1990, pp. 35-73.

[60] I. Poulakakis, J. A. Smith, and M. Buehler, "Experimentally validated bounding models for the scout ii quadrupedal robot," in ICRA, 2004, pp. $2595-2600$.

[61] G. Haynes and A. Rizzi, "Gait regulation and feedback on a robotic climbing hexapod," in Proceedings of Robotics: Science and Systems, Philadelphia, USA, August 2006.

[62] S. Revzen and J. M. Guckenheimer, "Estimating the phase of synchronized oscillators," Physical Review E, vol. 78, no. 5, p. 51907, 2008.

[63] G. A. Lynch, L. Rome, and D. E. Koditschek, "Sprawl angle in simplied models of vertical climbing: implications for robots and roaches," in Proceedings of the 2010 International Conference on Applied Bionics and Biomechanics, Venice, Italy, October 2011. 